DRAFT VERSION JUNE 24, 2021

Typeset using LATEX twocolumn style in AASTeX63

\title{
SN 2017fgc: A Fast-Expanding Type Ia Supernova Exploded in Massive Shell Galaxy NGC 474
}

\author{
Xiangyun Zeng,,${ }^{1,2}$ Xiaofeng Wang,,${ }^{3,4}$ Ali Esamdin, ${ }^{1}$ Craig Pellegrino,,${ }^{5,6}$ Jamison Burke, ${ }^{6,5}$ Benjamin E. Stahl, ${ }^{7,8,9}$ \\ WeiKang Zheng ${ }^{7}$ Alexei V. Filippenko, $,{ }^{7}, 10$ D. Andrew Howell,${ }^{6,5}$ D. J. Sand, ${ }^{11}$ Stefano Valenti, ${ }^{12}$ Jun Mo, ${ }^{3}$ Gaobo Xi, ${ }^{3}$

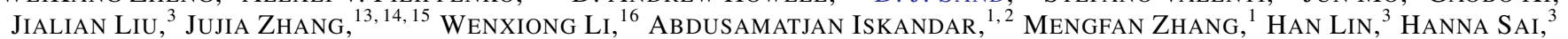 \\ Danfeng Xiang, ${ }^{3}$ Peng Wei, ${ }^{1}$ Tianmeng Zhang,${ }^{17}$ D. E. Reichart, ${ }^{18}$ Thomas G. Brink, ${ }^{7}$ Curtis McCully, ${ }^{6,5}$ \\ Daichi Hiramatsu, ${ }^{6,5}$ Griffin Hosseinzadeh, ${ }^{19,20,5}$ Benjamin T. JefFers, ${ }^{7}$ Timothy W. Ross, ${ }^{7}$ Samantha Stegman, ${ }^{7,21}$ \\ LifAN WANG, ${ }^{22}$ JiCHENG ZHANG, $^{3}$ AND SHUO MA ${ }^{1,2}$ \\ ${ }^{1}$ Xinjiang Astronomical Observatory, Chinese Academy of Sciences, Urumqi, Xinjiang 830011, People's Republic of China \\ ${ }^{2}$ School of Astronomy and Space Science, University of Chinese Academy of Sciences, Beijing 100049, People's Republic of China \\ ${ }^{3}$ Physics Department and Tsinghua Center for Astrophysics (THCA), Tsinghua University, Beijing, 100084, People's Republic of China \\ ${ }^{4}$ Beijing Planetarium, Beijing Academy of Science of Technology, Beijing 100044, People's Republic of China \\ ${ }^{5}$ Department of Physics, University of California, Santa Barbara, CA 93106-9530, USA \\ ${ }^{6}$ Las Cumbres Observatory, 6740 Cortona Drive Suite 102, Goleta, CA 93117-5575, USA \\ ${ }^{7}$ Department of Astronomy, University of California, Berkeley, CA 94720-3411, USA \\ ${ }^{8}$ Department of Physics, University of California, Berkeley, CA 94720-7300, USA \\ ${ }^{9}$ Marc J. Staley Graduate Fellow \\ ${ }^{10}$ Miller Senior Fellow, Miller Institute for Basic Research in Science, University of California, Berkeley, CA 94720, USA \\ ${ }^{11}$ Steward Observatory, University of Arizona, 933 North Cherry Avenue, Rm. N204, Tucson, AZ 85721-0065, USA \\ ${ }^{12}$ Department of Physics, University of California, Davis, CA 95616, USA \\ ${ }^{13}$ Yunnan Observatories (YNAO), Chinese Academy of Sciences, Kunming 650216, People's Republic of China \\ ${ }^{14}$ Key Laboratory for the Structure and Evolution of Celestial Objects, Chinese Academy of Sciences, Kunming 650216, People's Republic of China \\ ${ }^{15}$ Center for Astronomical Mega-Science, Chinese Academy of Sciences, 20A Datun Road, Chaoyang District, Beijing, 100012, People's Republic of China \\ ${ }^{16}$ The School of Physics and Astronomy, Tel Aviv University, Tel Aviv 69978, Israel \\ ${ }^{17}$ Key Laboratory of Optical Astronomy, National Astronomical Observatories, Chinese Academy of Sciences, Beijing 100012, People's Republic of China \\ ${ }^{18}$ Department of Physics and Astronomy, University of North Carolina at Chapel Hill, Chapel Hill, NC 27599, USA \\ ${ }^{19}$ Center for Astrophysics, Harvard \& Smithsonian, 60 Garden Street, Cambridge, MA 02138-1516, USA \\ ${ }^{20}$ Las Cumbres Observatory, 6740 Cortona Drive Suite 102, Goleta, CA 9311-5575, USA \\ ${ }^{21}$ Department of Chemistry, University of Wisconsin, Madison, WI 53706, USA \\ ${ }^{22}$ George P. and Cynthia Woods Mitchell Institute for Fundamental Physics \& Astronomy, Texas A\&M University, Department of Physics and Astronomy, 4242 \\ TAMU, College Station, TX 77843, USA
}

\begin{abstract}
We present extensive optical photometric and spectroscopic observations of the high-velocity (HV) Type Ia supernova (SN Ia) $2017 \mathrm{fgc}$, covering the phase from $\sim 12 \mathrm{~d}$ before to $\sim 389 \mathrm{~d}$ after maximum brightness. SN $2017 \mathrm{fgc}$ is similar to normal SNe Ia, with an absolute peak magnitude of $M_{\max }^{B} \approx-19.32 \pm 0.13 \mathrm{mag}$ and a post-peak decline of $\Delta m_{15}(B)=1.05 \pm 0.07 \mathrm{mag}$. Its peak bolometric luminosity is derived as $(1.32 \pm 0.13) \times 10^{43} \mathrm{erg} \mathrm{s}^{-1}$, corresponding to a ${ }^{56} \mathrm{Ni}$ mass of $0.51 \pm 0.03 M_{\odot}$. The light curves of SN $2017 \mathrm{fgc}$ are found to exhibit excess emission in the $U B V$ bands in the early nebular phase and pronounced secondary shoulder/maximum features in the $\operatorname{Rr} I i$ bands. Its spectral evolution is similar to that of $\mathrm{HV}$ $\mathrm{SNe}$ Ia, with a maximum-light Si II velocity of $15,000 \pm 150 \mathrm{~km} \mathrm{~s}^{-1}$ and a post-peak velocity gradient of $\sim 120 \pm 10 \mathrm{~km} \mathrm{~s}^{-1} \mathrm{~d}^{-1}$. The Fe II and Mg II lines blended near $4300 \AA$ and the Fe II, Si II, and Fe III lines blended near $4800 \AA$ are obviously stronger than those of normal SNe Ia. Inspecting a large sample reveals that the strength of the two blends in the spectra, and the secondary peak in the $i / r$-band light curves, are found to be positively correlated with the maximum-light Si II velocity. Such correlations indicate that HV SNe Ia may experience more complete burning in the ejecta and/or that their progenitors have higher metallicity. Examining
\end{abstract}

wang_xf@mail.tsinghua.edu.cn

aliyi@xao.ac.cn 
the birthplace environment of SN $2017 \mathrm{fgc}$ suggests that it likely arose from a stellar environment with young and high-metallicity populations.

Keywords: supernovae: individual: SN 2017fgc — supernovae: general: fast expanding

\section{INTRODUCTION}

Type Ia supernovae (SNe Ia) are widely believed to originate from thermonuclear runaway explosions of carbonoxygen (CO) white dwarfs (WDs) in binary systems (e.g., Nomoto et al. 1997; Hillebrandt \& Niemeyer 2000; Maoz et al. 2014; Livio \& Mazzali 2018; Soker 2019), and they have a typical absolute $V$-band peak magnitude of $\sim$ -19 mag (e.g., Phillips 1993; Perlmutter et al. 1999; Wang et al. 2006). One of the main applications of SNe Ia is that they can be utilized as extragalactic distance indicators (e.g., Riess et al. 1996; Wang et al. 2005; Guy et al. 2005; Howell et al. 2006; Howell 2011; Burns et al. 2018; Scolnic et al. 2018), leading to the discovery of the accelerating expansion of the Universe (Riess et al. 1998; Perlmutter et al. 1999).

Two prevailing ideas for the progenitor systems are the double-degenerate (DD) scenario (Webbink 1984; Iben \& Tutukov 1984) and the single-degenerate (SD) scenario (Whelan \& Iben 1973; Nomoto et al. 1984; Podsiadlowski et al. 2008). The former scenario involves the dynamical merger of two WDs with an accretion phase (Rasio \& Shapiro 1994; Yoon et al. 2007; Pakmor et al. 2012; Sato et al. 2015) or the violent/third-body-induced collision of a binary WD (Thompson 2011; Pakmor et al. 2012; Raskin et al. 2014; Sato et al. 2015), while explosion in the latter case is triggered by accretion onto a WD from its nondegenerate companion (Whelan \& Iben 1973; Webbink 1984; Iben \& Tutukov 1984). However, details of the progenitor systems and explosion mechanisms of SNe Ia are still controversial (Wang et al. 2013; Maoz et al. 2014; Jha et al. 2019; Han et al. 2020). Some tentative evidence presented for the absence of companion stars in some SNe Ia favors the DD scenario (González Hernández et al. 2012; Schaefer \& Pagnotta 2012; Olling et al. 2015; Tucker et al. 2019), while the possible detections of circumstellar material (CSM) support the SD scenario for at least a portion of SNe Ia (Hamuy et al. 2003; Wang et al. 2004; Aldering et al. 2006; Pastorello et al. 2007; Blondin et al. 2009; Sternberg et al. 2011a; Taddia et al. 2012; Dilday et al. 2012; Silverman et al. 2013; Bochenek et al. 2018; Wang et al. 2019b), though some theoretical studies show that the CSM could be also produced in the DD scenario (Raskin \& Kasen 2013; Shen et al. 2013; Levanon \& Soker 2017).

Observations show that $\sim 70 \%$ of SNe Ia are members of the spectroscopically normal subclass (Branch et al. 1993; Li et al. 2011), while the others are classified as peculiar, including the overluminous SN 1991T-like (Filippenko et al. 1992a; Ruiz-Lapuente et al. 1992; Filippenko 1997), the sublumi- nous SN 1991bg-like (Filippenko et al. 1992b; Leibundgut et al. 1993), and the low-maximum-light velocity with low luminosity SN Iax 2002cx-like (Filippenko 2003; Li et al. 2003; Foley et al. 2013). Benetti et al. (2005) divided normal SNe Ia into three subclasses: high velocity gradient (HVG), low velocity gradient (LVG), and faint, according to the temporal velocity gradient of the Si II line. Based on the equivalent width (EW) of Si II $\lambda 6355$ and Si II $\lambda 5972$ measured at $B$-band maximum, Branch et al. (2006) proposed that $\mathrm{SNe}$ Ia could be classified into core normal $(\mathrm{CN})$, broad line (BL), cool (CL), and shallow silicon (SS) subgroups. According to the Si II $\lambda 6355$ velocity measured at $B$-band maximum, Wang et al. (2009a) suggested that the "Branch-normal" SNe Ia could be classified into normalvelocity (i.e., $v<12,000 \mathrm{~km} \mathrm{~s}^{-1}$; NV) and high-velocity (i.e., $v \geq 12,000 \mathrm{~km} \mathrm{~s}^{-1}$; HV) subclasses. Although various classification schemes have been proposed to classify SNe Ia, there are overlaps between different classifications as demonstrated by different samples (Blondin et al. 2012; Silverman et al. 2012b). For example, HV SNe Ia are usually found to have large velocity gradient and broad line profiles (Barbon et al. 1989; Benetti et al. 2004; Wang et al. 2009a; Yamanaka et al. 2009; Silverman \& Filippenko 2012). Moreover, after the analysis of the birthplace environments of SNe Ia in their host galaxies, Wang et al. (2013) suggest that the HV and NV subclasses of SNe Ia may come from progenitor systems with different metallicities.

A recent study by Pan et al. (2015) and Li et al. (2021) suggest that $\mathrm{HV} \mathrm{SNe}$ Ia tend to reside in massive galaxies and likely have metal-rich progenitor environments. Through an investigation of a large set of the host galaxies, Pan (2020) found that HV SNe might arise in massive host galaxies with metal-rich environments. Based on an analysis of $\mathrm{Na} \mathrm{I}$ absorption lines in the spectra and the late-time light curves in the $B$ and $V$ bands, Wang et al. (2019b) show that HV SNe Ia likely have more abundant circumstellar dust around their progenitors, and they may originate from progenitor systems with nondegenerate companions.

SN $2017 \mathrm{fgc}$ is a fast-expanding SN Ia that exploded in the nearby shell galaxy NGC 474 at a distance of $29.51 \pm 2.09$ Mpc (Cappellari et al. 2011). Note that the host is a lenticular galaxy with prominent gas shell and bridge structures at its outskirts, suggestive of the merging process (Lim et al. 2017; Alabi et al. 2020; Fensch et al. 2020). Burgaz et al. (2021) studied the photometric properties of this SN and proposed the presence of a prominent $R I$-band secondary shoulder/maximum. Here we present extensive photometric and 
spectroscopic observations of SN $2017 \mathrm{fgc}$, and we report an additional discrepancy between the NV and HV subclasses in a well-observed sample of SNe Ia.

In this paper, the optical observations and data reduction are presented in Section 2. Section 3 discusses the light curve, color curves, and quasibolometric light curve, while Section 4 presents the spectroscopic evolution. The properties of SN $2017 \mathrm{fgc}$ and its host galaxy are discussed in Section 5. We summarize in Section 6.

\section{OBSERVATIONS AND DATA REDUCTION}

\subsection{Discovery and Host Galaxy}

SN 2017fgc was discovered at $\alpha=01^{h} 20^{m} 14^{s} .440$, $\delta=03^{\circ} 24^{\prime} 09^{\prime \prime} .96$ (J2000) on 2017 July 9.29 (UT dates are adopted throughout this paper) by the Distance Less Than 40 Mpc (DLT40; Tartaglia et al. 2018a) survey at $r=$ $17.32 \mathrm{mag}$ (Valenti et al. 2017b). At that time, the DLT40 survey operated with a $\sim 24 \mathrm{hr}$ cadence, and the last nondetection of the SN was on 2018 July 8.29 with a limiting magnitude of $r \approx 19.5 \mathrm{mag}$. A spectrum taken $\sim 1.4 \mathrm{hr}$ after the discovery classified it as a normal SN Ia (Valenti et al. 2017a). The host galaxy of SN $2017 \mathrm{fgc}$ is NGC 474 with a redshift of $z=0.00772 \pm 0.00002$ (HernándezToledo et al. 2011), which corresponds to a distance modulus of $\mu=32.51 \pm 0.11$ mag assuming a Hubble constant of $73.5 \mathrm{~km} \mathrm{~s}^{-1} \mathrm{Mpc}^{-1}$ (Riess et al. 2018).

\subsection{Photometry}

Our photometric observations of SN 2017fgc were obtained with several telescopes, including the $0.8 \mathrm{~m}$ TsinghuaNAOC telescope (TNT; Huang et al. 2012), the Las Cumbres Observatory (LCO) Telescope network (Brown et al. 2010), the $0.76 \mathrm{~m}$ Katzman Automatic Imaging Telescope (KAIT) at Lick Observatory (Filippenko et al. 1999, 2001), and the $1 \mathrm{~m}$ Nickel reflector ${ }^{1}$ at Lick Observatory. The TNT and Nickel telescope monitored SN 2017fgc in the $B V R I$ bands, KAIT observed it in the $B V R I$ and Clear bands, and the LCO $1 \mathrm{~m}$ telescopes sampled the light curves in the $U B V g r i$ bands. Figure 1 shows color images of SN $2017 \mathrm{fgc}$ synthesized from observations in gri bands. The left panel shows a color image is synthesized from $\mathrm{CFHT}^{2}$ observations in $g r$ bands processed by J.-C. Cuillandre and G. Anselmi, based on data from the MATLAS program (Duc et al. 2015), while the right panel shows a color image systhesized from TNT observations.

For photometric images obtained from the LCO during the Global Supernova Project, lcogtsnpipe (Valenti et al. 2016) was employed for image reduction. The point-spread

\footnotetext{
${ }^{1}$ https://www.ucolick.org/public/telescopes/nickel.html

2 https://www.cfht.hawaii.edu/HawaiianStarlight/images.html
}

functon (PSF) implemented in Photutils (Bradley et al. 2020) was utilized to extract instrumental magnitudes of SN 2017 fgc from images obtained by LCO. Photometric images from KAIT and Nickel were reduced with LOSSPhotPypeline $^{3}$ (Stahl et al. 2019, 2020a). The DLT40 images were reduced using a dedicated difference-imaging pipeline (Tartaglia et al. 2018b) which calibrates the Open filter data to the $r$ band utilizing the AAVSO Photometric All-Sky Survey $\left(\mathrm{APASS}^{4}\right.$ ) catalog. PSF photometry was performed on the difference images. As SN $2017 \mathrm{fgc}$ was located far away ( 18.90 $\pm 0.01 \mathrm{kpc}$ ) from the center of its host galaxy, we did not apply image-subtraction techniques for the photometry.

The color-transformation method introduced by Jordi et al. (2006) was employed to convert gri magnitudes from SDSS Dr12 (Alam et al. 2015) to Landolt $U B V R I$ (Landolt 1992) magnitudes. The local standard stars with SDSS gri magnitudes and the transformed $U B V R I$ magnitudes are listed in Table 1. The instrumental magnitudes obtained from TNT and KAIT in $B V R I$ are calibrated to the Johnson $U B V R I$ system (Landolt 1992; Stetson 2000). The unfiltered magnitudes from KAIT are calibrated to the standard Landolt $R$ band magnitudes, with a typical uncertainty of about $0.2-$ $0.3 \mathrm{mag}$ (Li et al. 2003; Zheng et al. 2017b). The LCO instrumental magnitudes in $U B V$ are calibrated to the Johnson system (Landolt 1992; Stetson 2000), while the gri magnitudes are calibrated using the SDSS Dr12 catalog (Alam et al. 2015). The final light curves are shown in Figure 2, and the flux-calibrated magnitudes are listed in Table 2.

\subsection{Spectroscopy}

A total of 38 low-resolution spectra were obtained for SN 2017 fgc with different instruments, including the FLOYDS spectrographs mounted on the LCO-2 m Faulkes Telescope North and South (FTN and FTS; Brown et al. 2013; Sand et al. 2011), the BFOSC mounted on the Xinglong $2.16 \mathrm{~m}$ telescope (XLT; Jiang et al. 1999; Zhang et al. 2016a; Fan et al. 2016), the YFOSC on the Lijiang $2.4 \mathrm{~m}$ telescope (LJT; Chen et al. 2001; Wang et al. 2019a) of Yunnan Astronomical Observatories, and the Kast spectrograph on the Lick $3 \mathrm{~m}$ Shane telescope (Filippenko et al. 1986; Vogt 1987; Stahl et al. 2020b). Three additional spectra of SN $2017 \mathrm{fgc}$ were obtained with XSHOOTER (Vernet et al. 2011) mounted on the ESO Very Large Telescope (VLT), at $t \approx+149.7 \mathrm{~d}$, $+383.9 \mathrm{~d}$, and 388.9 d during ESO programs 0101.D-0242(A) and 0101.D-0443(A) (Graur et al. 2020). The journal of spectroscopic observations is presented in Table 3.

\footnotetext{
3 https://github.com/benstahl92/LOSSPhotPypeline

${ }^{4}$ https://www.aavso.org/apass
} 

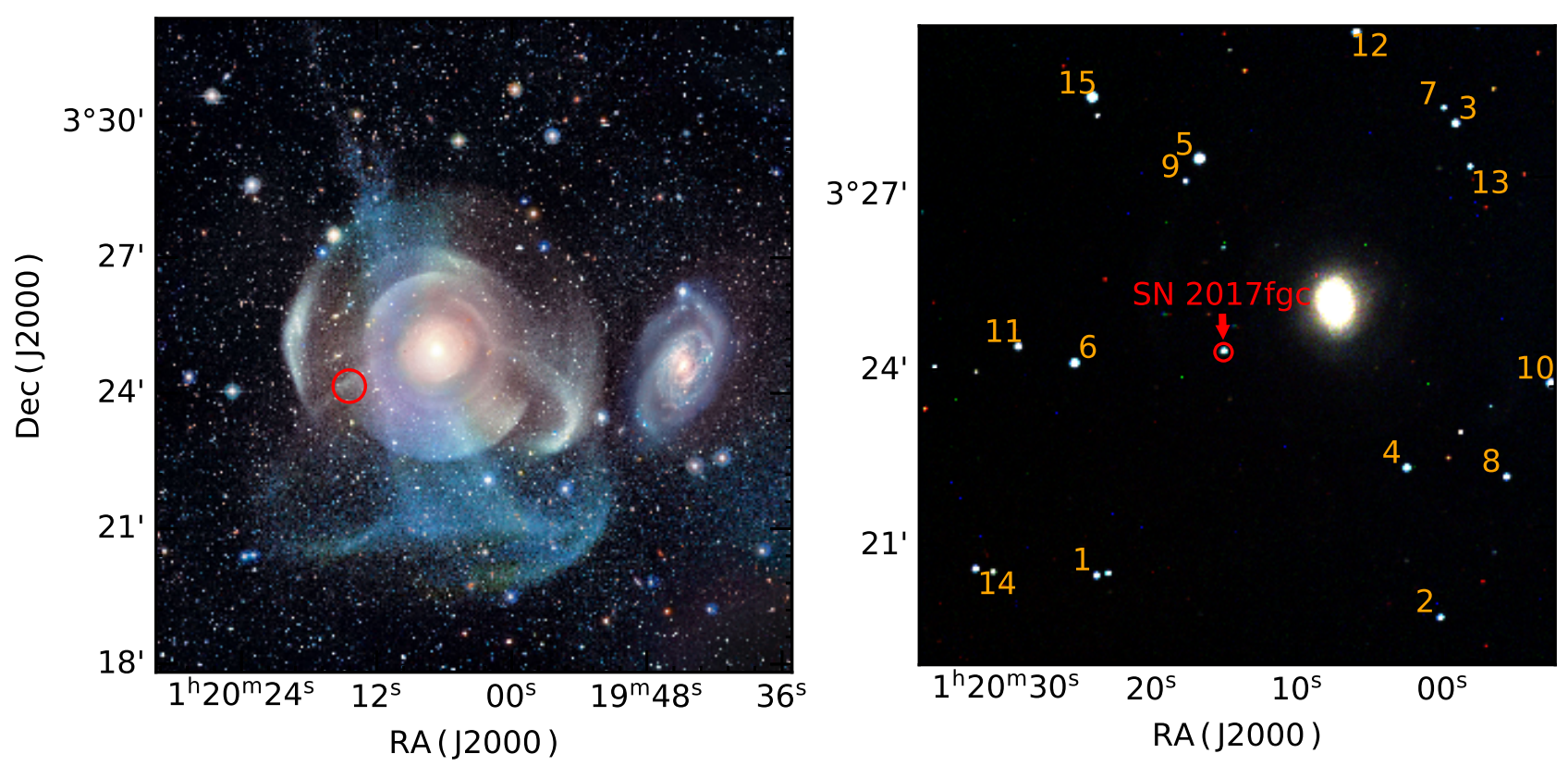

Figure 1. The left panel shows a color image which is synthesized from observations in $g r$ bands obtained by the CFHT before the discovery of SN $2017 \mathrm{fgc}$. The red circle marks the position of SN $2017 \mathrm{fgc}$ and the gas bridge can be clearly seen here. The right panel shows a color image synthesized from TNT observations in the gri bands after the explosion of SN $2017 \mathrm{fgc}$, and the SN is marked with a red circle while the reference stars are labeled by numbers.

Standard IRAF ${ }^{5}$ routines were used to reduce the spectra. Spectrophotometric standard stars observed at an airmass comparable to the target on the same night were used for flux calibration. The extinction curves at various observatories were utilized to correct for atmospheric extinction, and spectra of the standard stars were used to eliminate the telluric absorption lines.

\section{LIGHT CURVES}

\subsection{Optical Light Curves}

Figure 2 shows the optical light curves of SN $2017 \mathrm{fgc}$, covering the phases from about two weeks before to over $200 \mathrm{~d}$ after $B$-band maximum light. Overall, they are similar to those of normal SNe Ia, characterized by a prominent shoulder or secondary maximum in the $R / r$ and $I / i$ bands. A small shoulder may also be detectable in the $V$ and Clear bands. Applying a polynomial fit to the $B$-band light curves around maximum light yields a peak value of $13.07 \pm 0.11 \mathrm{mag}$ on MJD = 57959.4 (2017 July 25.4 UT). The $V$-band light curve reached its peak of $12.91 \pm 0.07 \mathrm{mag}$ on MJD $=57962.5, \sim 3.1 \mathrm{~d}$ after the $B$-band peak. The postpeak $B$-band decline rate $\Delta m_{15}(B)$ is measured as $1.05 \pm 0.07 \mathrm{mag}$, and the color stretch (Burns et al. 2011, 2014) is determined to be $s_{B V}=1.19 \pm 0.03$.

\footnotetext{
${ }^{5}$ IRAF is distributed by NOAO, which is operated by AURA, Inc., under cooperative agreement with the U.S. National Science Foundation (NSF).
}

In Figures 3 and 4 the $U B V g$ and $\operatorname{Rr} I i$ light curves of SN $2017 \mathrm{fgc}$ are compared with those of well-observed normal SNe Ia having similar $\Delta m_{15}(B)$, including SNe 2002bo (Krisciunas et al. 2004; Benetti et al. 2004), 2003du (Stanishev et al. 2007), 2005cf (Wang et al. 2009b), 2006X (Wang et al. 2008b), 2007af (Stritzinger et al. 2011), 2007le (Ganeshalingam et al. 2010; Hicken et al. 2012), 2009ig (Marion et al. 2013), 2011fe (Munari et al. 2013; Zhang et al. 2016b), 2013gs (Zhang et al. 2019), 2017hpa (Zeng et al. 2021), and 2018oh ( $\mathrm{Li}$ et al. 2019). One can see that the light curves of SN $2017 \mathrm{fgc}$ are generally similar to those of comparison $\mathrm{SNe}$ Ia near the $B$-band maximum. We notice, however, that SN $2017 \mathrm{fgc}$ seems to have brighter tails in both $U$ and $B$ relative to those of NV SNe Ia, consistent with the tendency that HV SNe Ia have flatter evolution at $t \approx 1-3$ months after maximum light (e.g., Wang et al. 2008b, 2019b). For example, the magnitude decline measured within $60 \mathrm{~d}$ after peak brightness is $3.57 \pm 0.08 \mathrm{mag}$ in $U$ and $2.96 \pm 0.08 \mathrm{mag}$ in $B$, similar to the evolution of the well-known HV SN 2006X. Figure 4 shows a comparison of the RrIi light curves. One can see that SN $2017 \mathrm{fgc}$ has a more significant secondary shoulder or peak in the $R / r$ and $I / i$ bands than the normal counterparts.

\subsection{Reddening}

The line-of-sight Galactic extinction of SN $2017 \mathrm{fgc}$ is estimated to be $A_{V}=0.094 \mathrm{mag}$ (Schlegel et al. 1998; Schlafly \& Finkbeiner 2011). Adopting $R_{V}=3.1$ (Cardelli et al. 


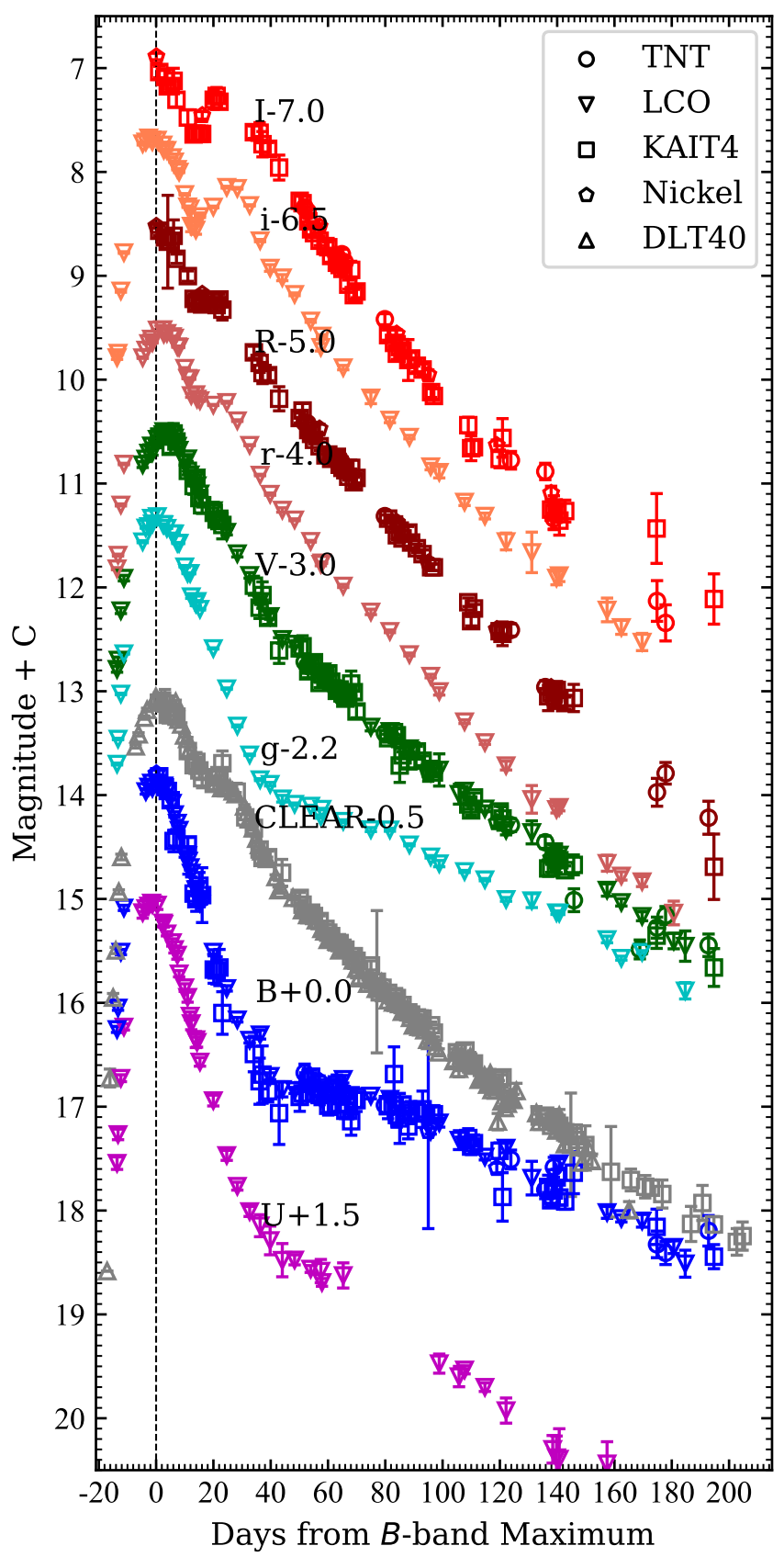

Figure 2. The optical light curves of SN 2017 fgc. The different colors represent different observation bands, while data from different telescopes/instruments are indicated with different symbols. The vertical dashed line represents the time of the $B$-band maximum, relative to which all times are given in this paper. The light curves have been shifted vertically for clarity.

1989), this corresponds to a color excess of $E(B-V)_{\mathrm{Gal}}=$ 0.030 mag. After removal of the Galatic extinction, the $B-V$ color of SN $2017 \mathrm{fgc}$ is estimated to be $-0.03 \pm 0.02 \mathrm{mag}$ at $t=0 \mathrm{~d}$ and $1.62 \pm 0.10 \mathrm{mag}$ at $t=+35 \mathrm{~d}$ relative to the $B$-band maximum, consistent with typical values of normal SNe Ia (Phillips et al. 1999; Wang et al. 2009a).
SuperNovae in object-oriented Python (SNooPy2; Burns et al. 2011, 2014) has also been employed to fit the multiband light curves of SN 2017fgc, and the model fitting results are shown in Figure 5(a). We adopt the $E B V$ model with sttype and estimate the reddening due to the host galaxy as $E(B-V)_{\text {host }}=0.17 \pm 0.07$ mag, suggesting an insignificant host-galaxy reddening for SN 2017fgc. This is consistent with the absence of $\mathrm{Na}$ I D absorption lines in the optical spectra of SN $2017 \mathrm{fgc}$.

\subsection{Color Curves}

Figure 6 shows the color evolution of SN $2017 \mathrm{fgc}$ compared with that of several well-observed SNe Ia having similar $\Delta m_{15}(B)$. At early times, both the $B-V$ and $g-r$ color curves evolve toward the blue until reaching the blue peak at $t \approx 0 \mathrm{~d}$; then they evolve redward and reach the red peak at $t \approx 35 \mathrm{~d}$. The $B-V$ and $V-I$ color curves of SN $2017 \mathrm{fgc}$ show close resemblances to those of SN 2002bo, SN 2006X, and SN 2009ig, which all belong to the subclass of HV SN Ia (Krisciunas et al. 2004; Benetti et al. 2004; Wang et al. 2008b; Silverman \& Filippenko 2012; Hicken et al. 2012). However, none of the comparison SNe Ia look perfectly the same as SN $2017 \mathrm{fgc}$. While evolving redwards, the $g-r$ color curves of SN 2017fgc and other comparison SNe Ia show a clear jump at $t \sim 1$ week after maximum light, which is similar to that of the $V-R$ color curves at similar phase (Cartier et al. 2014; Gutiérrez et al. 2016; Zhang et al. 2019). Both the $V-I$ and $g-i$ color curves show an evolution from red to blue until $t \approx 15 \mathrm{~d}$; then they evolve redward and reach the red peak at $t \approx 35 \mathrm{~d}$. Like other HV SNe Ia, SN 2017fgc also exhibited an overall bluer $V-I$ color in comparison with the NV counterparts. The prominent Ca II near-infrared (NIR) absorption features shown in the spectra of HV SNe Ia are likely responsible for their bluer $V-I$ colors.

\subsection{First-Light Time}

The Distance Less Than 40 (DLT40) Mpc survey intensely observed SN2017fgc at extremely early times, using a clear band (calibrated to $R$-band). The photometric data are listed in Table 4. Both the expanding fireball model from Riess et al. (1999) and the broken power-law model from Zheng et al. (2018) are adopted to fit the early light curve of SN 2017 fgc (as shown in Figure 7). The observed data at $t \leq-$ 10 days from the $R$-band peak are adopted to fit the fireball model while those obtained at $t \leq+15$ days are used for the broken power-law model fitting, and the estimated firstlight time (FLT) of the light curve are $57941.1 \pm 0.3 \mathrm{~d}$ and $57941.7 \pm 0.7 \mathrm{~d}$, respectively. The results of these two estimations are in a good agreement. The average fitted firstlight time is estimated as $57941.4 \pm 0.4 \mathrm{~d}$, hence, the rise time of SN $2017 \mathrm{fgc}$ is estimated as $18.0 \pm 0.4 \mathrm{~d}$. The multicolor 


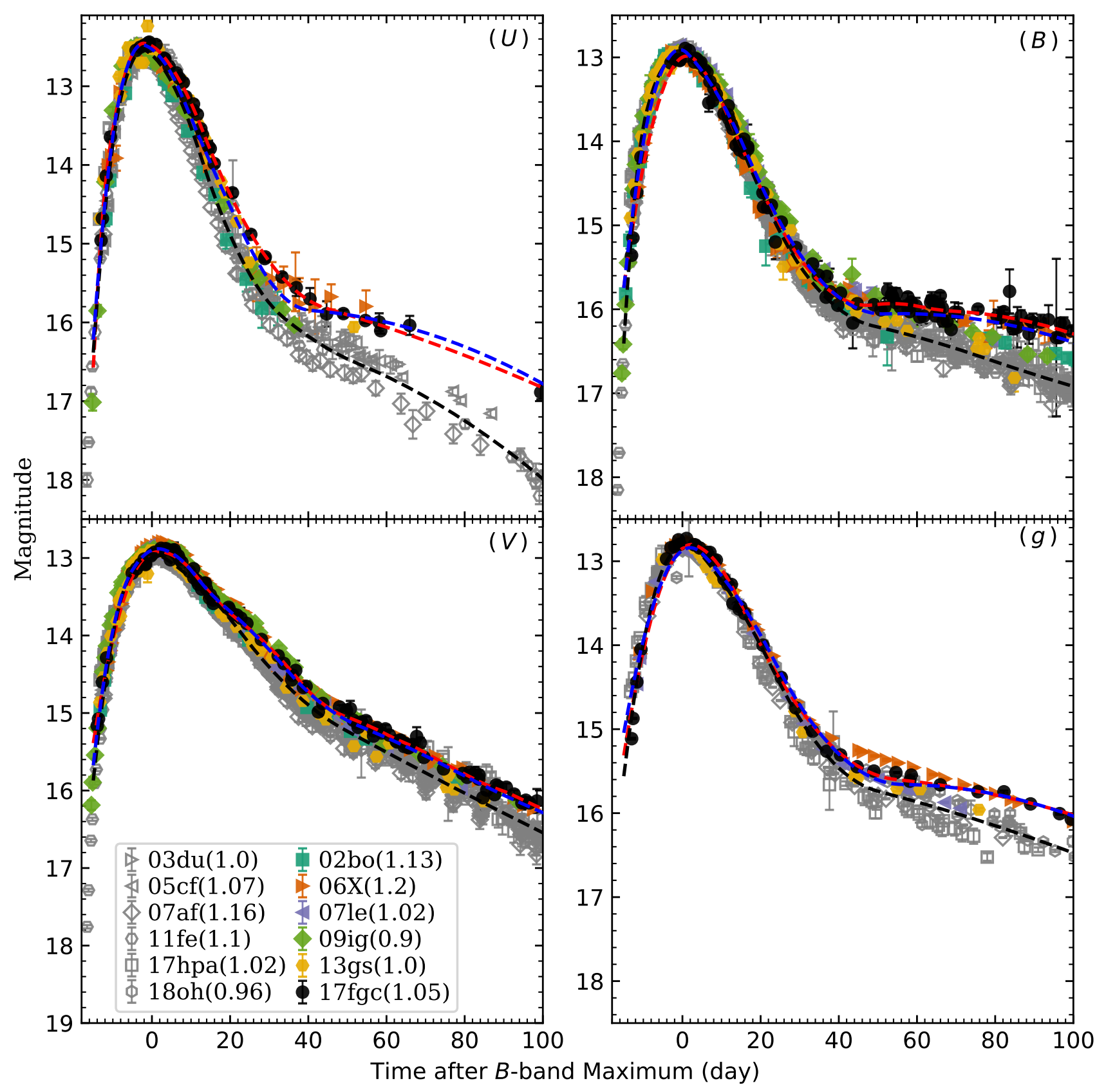

Figure 3. Comparison of the optical light curves (in the $U B V g$ bands) of SN 2017fgc with those of other well-observed SNe Ia having similar decline rates. The rest-frame light curves of the comparison SNe Ia have been normalized to match the peak magnitudes of SN $2017 \mathrm{fgc}$. No kcorrections have been applied to the light curves, as both SN 2017fgc and the comparison sample have a redshift less than 0.01. The NV SNe Ia are marked with open symbols while the HV SNe are shown with filled symbols. The black dashed lines represents the polynomial fit to the NV SNe Ia, while the blue dashed lines represents the case for HV SNe Ia. The red dashed lines represents the best-fit for the light curves of SN 2017 fgc. 


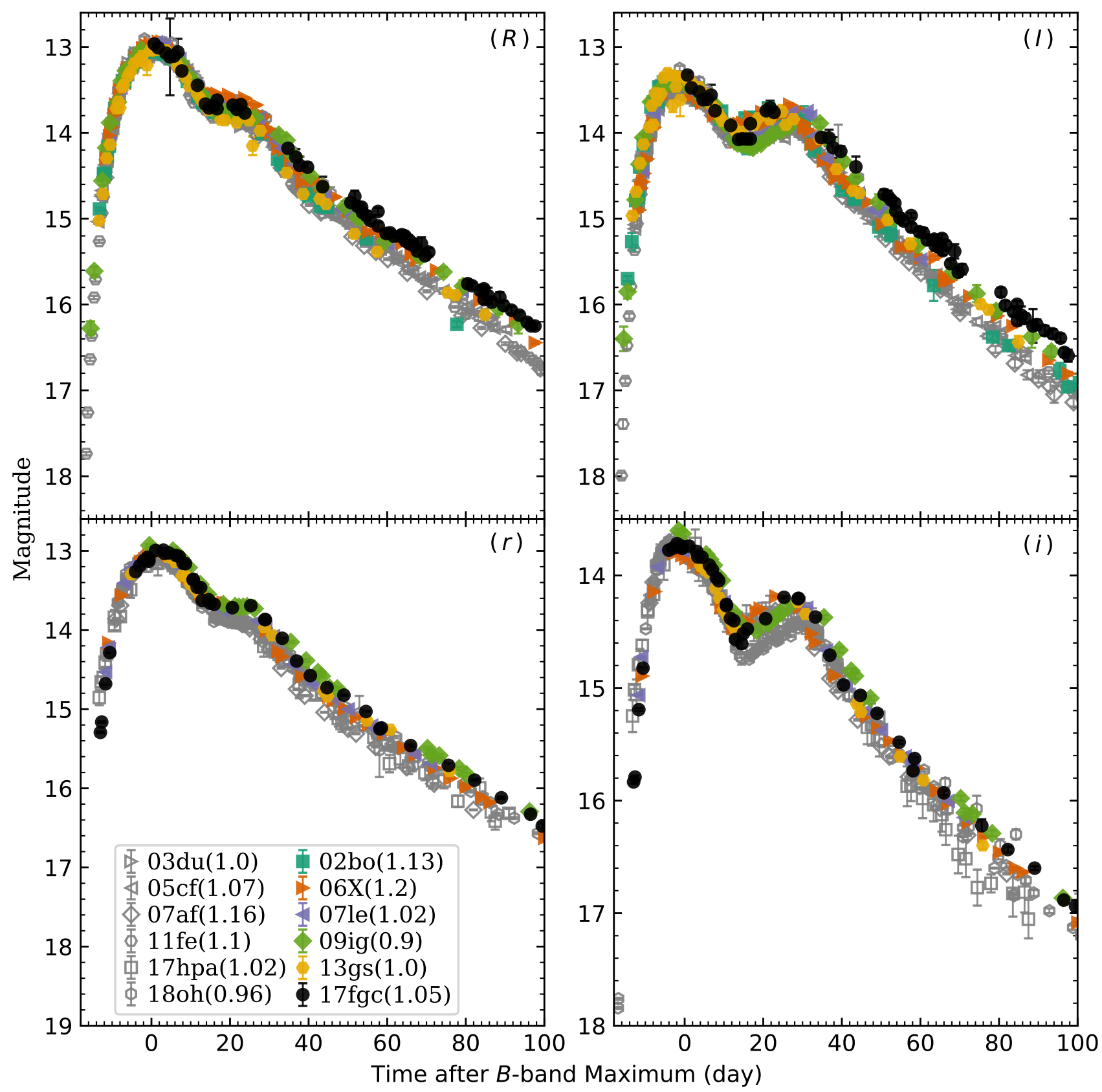

Figure 4. Comparison of the optical light curves (in the RrIi bands) of SN 2017fgc with those of other well-observed SNe Ia having similar decline rates. The light curves of the comparison SNe Ia have been normalized to match the peak magnitudes of SN $2017 \mathrm{fgc}$. The symbols are the same as in Figure 3.

observations of SN $2017 \mathrm{fgc}$ is conducted at $\sim 6$ days after the FLT, $\sim 12$ days before the $B$-band maximum. A flux excess seems to exsit for the first detection point, i.e., brighter than the fitting result by $\sim 0.23 \pm 0.07 \mathrm{mag}$ (see the bottom panel of Fig. 7). This excess flux detected in the early phase could be due to interaction of SN ejecta with nondegenerate companion (Kasen 2010), surrounding CSM (Gerardy et al. 2004; Piro \& Morozova 2016), or even the radioactive decay of nickel synthesized on the surface of the exploding WD
(Piro \& Morozova 2016; Noebauer et al. 2017). A further early-time light curves analysis including SN 2017fgc will be presented as part of a larger study (Burke et al. in preparation).

According to Zhang et al. (2010), the rise time of the $r$-band light curve of SNe Ia show an anticorrelation with $\Delta m_{15}(B)$ (see Figure 8). For given $\Delta m_{15}(B)$, the rise time of NV SNe Ia seems to be on average longer than that of the $\mathrm{HV}$ ones. A similar trend has been reported in other studies 


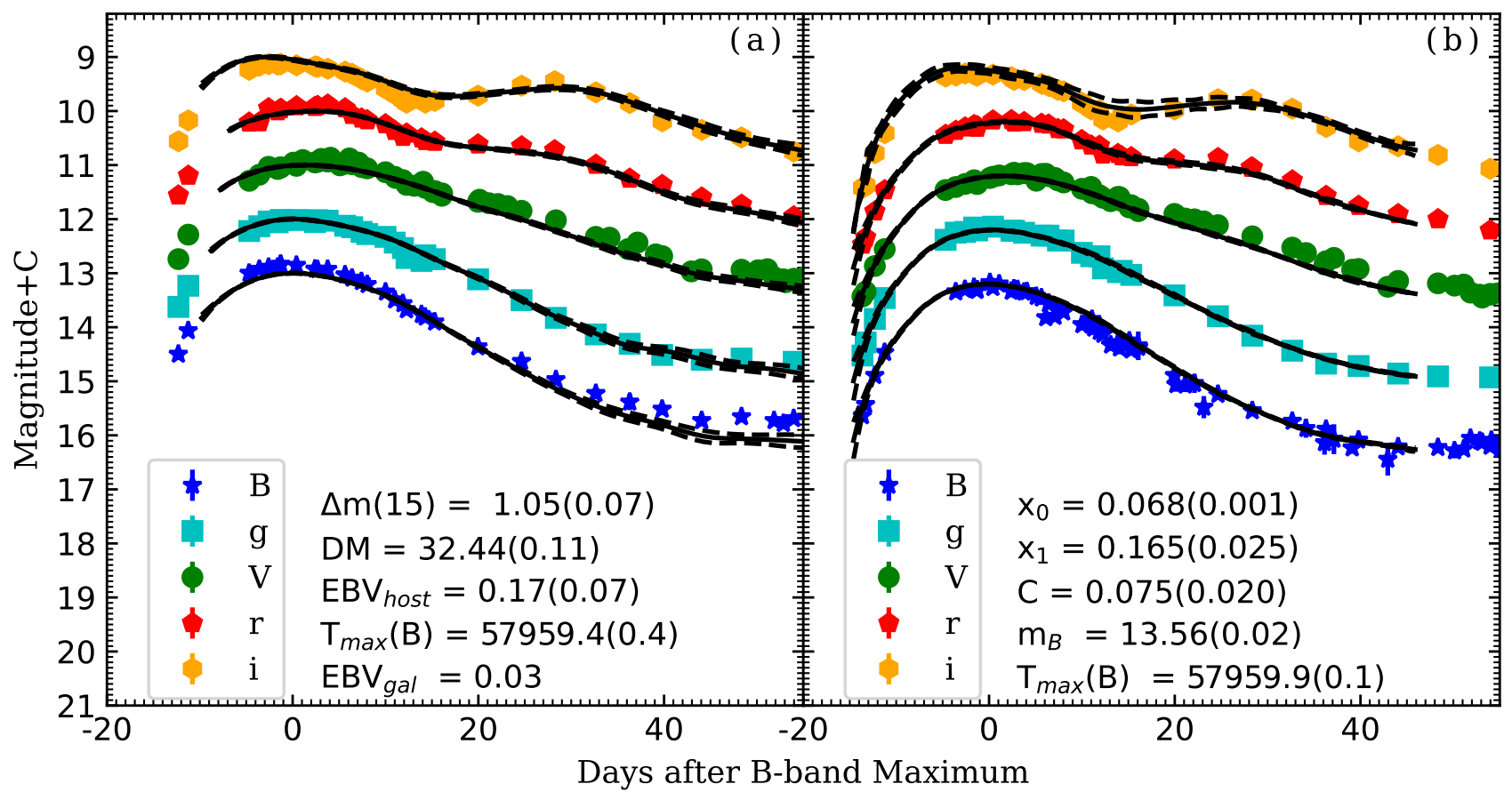

Figure 5. Best-fit light-curve model from SNooPy2 (left panel) and SALT 2.4 (right panel). The light curves are shifted vertically for clarity. The dashed lines represent the $1 \sigma$ uncertainty of the best-fit light-curve templates.

(e.g., Pignata et al. 2008; Ganeshalingam et al. 2011; Zheng et al. 2017a). As shown in Figure 8, SN 2017fgc also is in line with this trend. As the $R$-band and clear-band light curves have similar light curve shapes and magnitudes, we assume that they have similar rise time. The $R$-band rise time of SN $2017 \mathrm{fgc}$ is thus estimated as $18.0 \pm 0.4$ day by fitting its clear-band light curve. Given that the $R$-band light curve of SN 2017fgc reached its peak $(\sim 57961.3 \pm 0.2)$ at $\sim 1.9 \pm 0.4$ days later than that of the $B$-band $(\sim 57959.4 \pm 0.4)$, the $B$ band rise time can be thus inferred as $16.1 \pm 0.4 \mathrm{~d}$ assuming that the SN photons in these two bands diffuse out simultaneously. The $B$-band rise time speculated for SN 2017fgc is also obviously shorter than the mean value of SNe Ia (Zheng et al. 2017a). Compared to those of NV SNe Ia, the relatively shorter rise time seen in $\mathrm{HV}$ ones might be related to that their ejecta become optically thin at a faster pace because of more rapid expansion (Zhang et al. 2010).

\subsection{Quasi-Bolometric Light Curve}

According to Tully et al. (2013), based on the Tully-Fisher relation, the distance modulus measured for the host galaxy NGC 474 is $32.35 \pm 0.14 \mathrm{mag}$. From fitting to the multicolor light curves of SN $2017 \mathrm{fgc}$, SNooPy2 gives an average $\mu=32.44 \pm 0.11 \mathrm{mag}$ (see left panel of Fig. 5), while SALT 2.4 (Guy et al. 2010; Betoule et al. 2014) gives $\mu=32.39 \pm 0.07 \mathrm{mag}$ (see right panel of Fig. 5). These three estimates agree well with each other. The average value of $32.39 \pm 0.06 \mathrm{mag}$ is thus adopted in the following analysis. Assuming $R_{V}=3.1$, the absolute $B$-band peak magnitude of SN $2017 \mathrm{fgc}$ is estimated to be $M_{\max }(B)=$ $-19.32 \pm 0.13 \mathrm{mag}$ after correcting for both Galactic and host-galaxy extinction, which agrees well with that of normal SNe Ia (i.e., $M_{\max }(B) \approx-19.3 \mathrm{mag}$ for an SN Ia with $\Delta m_{15}(B) \approx 1.1$ mag; Phillips et al. 1999; Wang et al. 2009a).

Following the procedure used for SN 2018oh (Li et al. 2019), SNooPy2 is employed to establish the spectral energy distribution (SED) and thus the quasibolometric light curve of SN $2017 \mathrm{fgc}$ based on the $U, B, g, V, R, r, I$, and $i$ light curves. According to Wang et al. (2009b) and Zhang et al. (2016b), the UV/optical ratio of SN 2005cf and SN 2011fe (Normal SNe Ia) are measured as 0.095 and 0.085 , while the NIR/optical ratio of them are measured as 0.058 and 0.059 , respectively. The converted ratios relative to the bolometric luminosity are 0.082 and 0.074 respectively for SN 2005cf and SN 2011fe in UV bands, while those ratios in NIR bands are 0.050 and 0.052, respectively. Based on the SED of SN 2009ig (Marion et al. 2013; Chakradhari et al. 2019) near the maximum light, the UV/NIR to bolometric ratio of this $\mathrm{HV} \mathrm{SN}$ Ia are estimated as $0.058 \pm 0.010$ and $0.054 \pm 0.019$, respectively. Assuming that the average ultraviolet (UV) and NIR contributions are $7 \%$ and $5 \%$ for SN 2017fgc, the maximum-light luminosity is estimated as $L_{\text {peak }}=(1.32 \pm 0.13) \times 10^{43} \mathrm{erg} \mathrm{s}^{-1}$, reached at $\sim 0.96 \mathrm{~d}$ prior to the $B$-band maximum. This peak luminosity is larger than that of SN 2011fe $\left(\sim 1.13 \times 10^{43} \mathrm{erg} \mathrm{s}^{-1}\right.$; Zhang et al. 2016 b) but smaller than that of SN 2018 oh $\left(\sim 1.49 \times 10^{43} \mathrm{erg}\right.$ $\mathrm{s}^{-1}$; Li et al. 2019). 

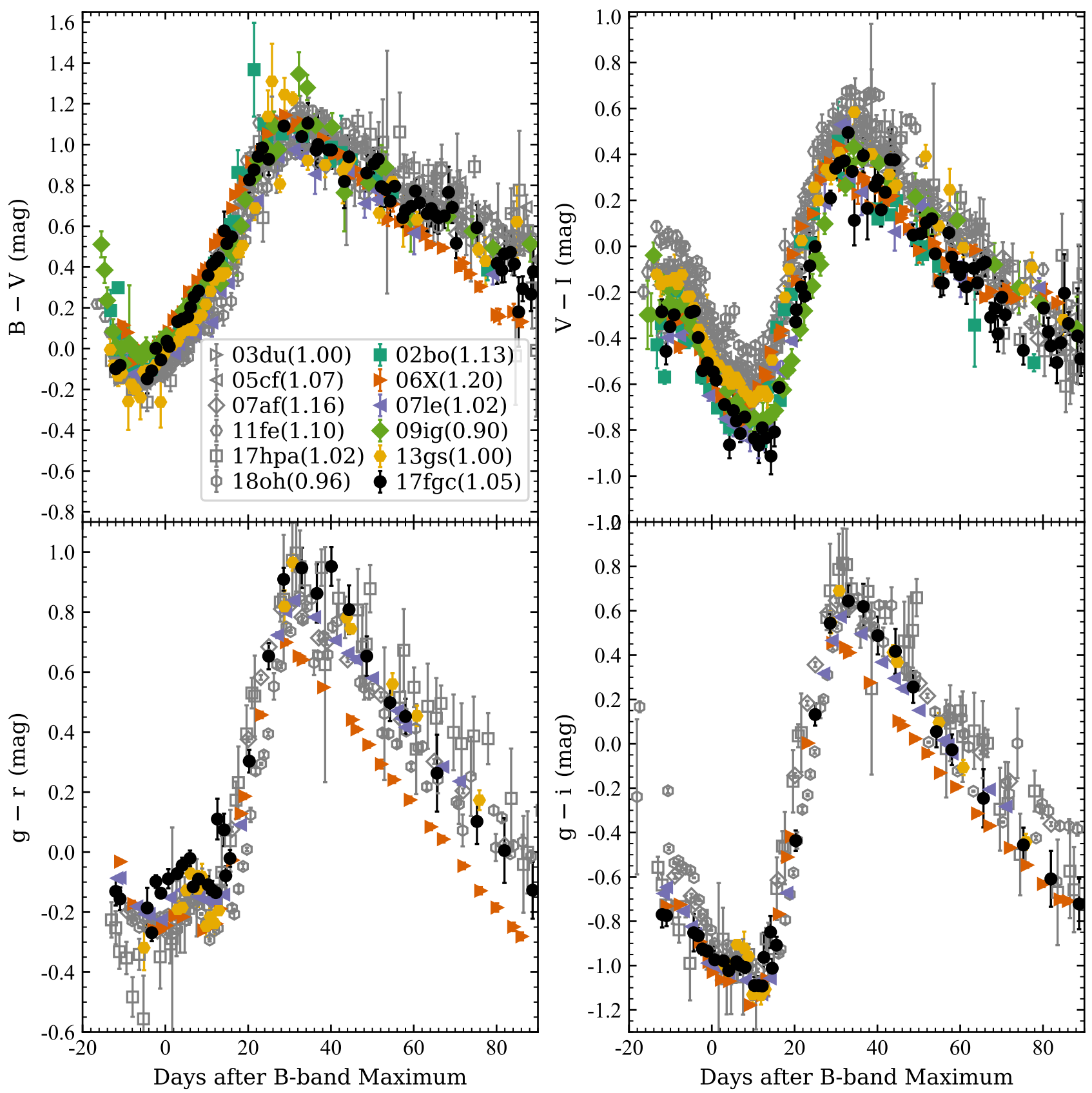

Figure 6. The $B-V, V-I, g-r$, and $g-i$ color curves of SN 2017fgc are compared with those of SNe 2002bo, 2003du, 2005cf, 2006X, 2007af, 2009ig, 2011fe, 2013gs, 2017hpa, and 2018oh. All color curves, including those of SN 2017fgc, have been dereddened using SNooPy2 (Burns et al. 2011, 2014).

The Minim Code (Chatzopoulos et al. 2013), utilizing a modified radiation diffusion model of Arnett (Arnett 1982; Chatzopoulos et al. 2012; Li et al. 2019), is used to fit the quasibolometric light curve with a constant-opacity approximation (see Figure 9). This fitting allows us to derive the following parameters, including the "first light" $t_{0}$, the model timescale of the light curve $t_{l c}$, the leaking timescale of gamma rays $t_{\gamma}$, and the radioactive ${ }^{56} \mathrm{Ni}$ ejecta mass $M_{\mathrm{Ni}}$ (see Chatzopoulos et al. 2012 for details), with the respective values being $57941.4 \pm 0.4,13.51 \pm 0.08 \mathrm{~d}, 38.59 \pm 2.28 \mathrm{~d}$, and $0.51 \pm 0.03 M_{\odot}$. The dark phase was presented as the delay between the explosion and the emergence of the radioactivity-powered light curve in several models of $\mathrm{SNe}$ Ia (Piro \& Nakar 2013). Assuming the initial diffusion wave reached the surface at $t=0$, the framework of Arnett model does not take a dark phase into account (Li et al. 2019). Piro \& Morozova (2016) proposed that the length of the dark phase could be less than 2 days. The first light $t_{0}$ from the 


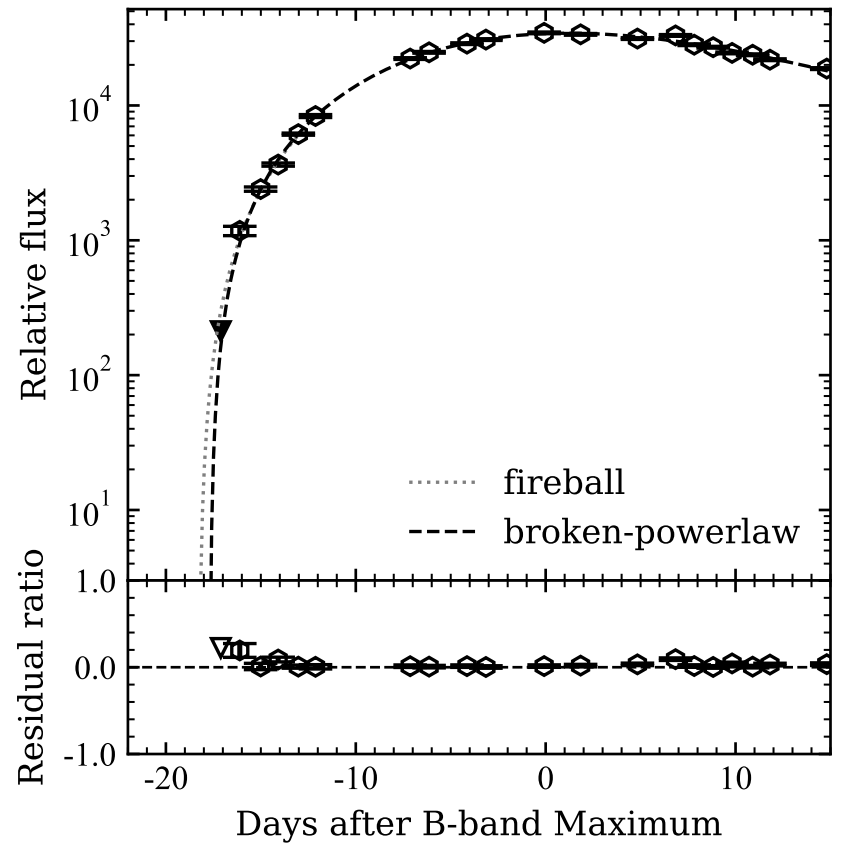

Figure 7. Fit to the observed clear band light curve using the ideal fireball model (Riess et al. 1999) and the analytic function introduced by Zheng et al. (2017a). The black triangle represents the earliest limit-magnitude from the DLT40 survey which is $\sim 0.6$ days after the fitted first-light time.

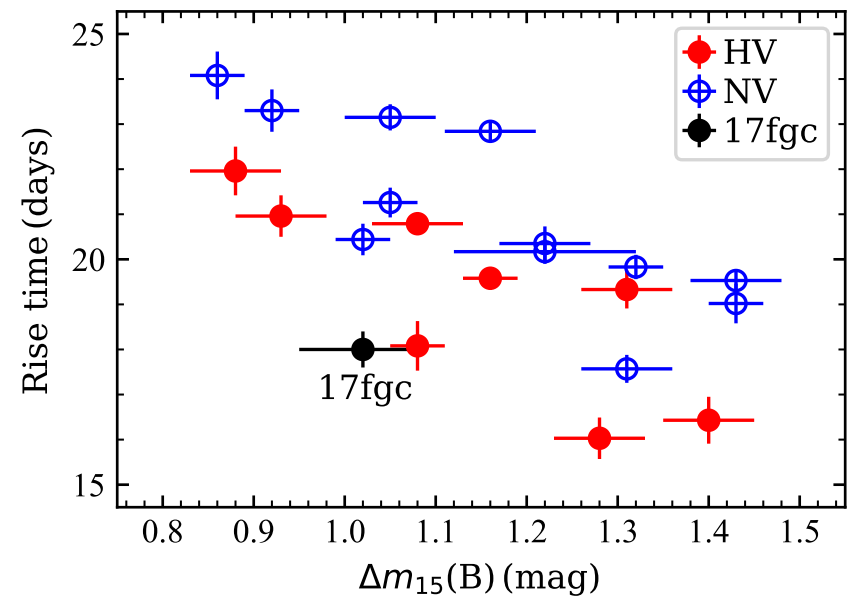

Figure 8. $R$-band rise times of several well-observed SNe Ia are plotted against the light curve decline rate $\Delta m_{15}(B)$. The data are taken from Zhang et al. (2010), and SN 2017fgc is overplotted in black dot. The HV SNe Ia are plotted as red dots while the NV ones are blue open circles.

Arnett model is $\sim 2.1 \mathrm{~d}$ later than that estimated from the fireball model or the broken-power-law model. Considering the model-dependent uncertainties in the estimation, we interpreted that the difference in the two estimated starting moments could be related to the dark phase in SN 2017fgc (Piro

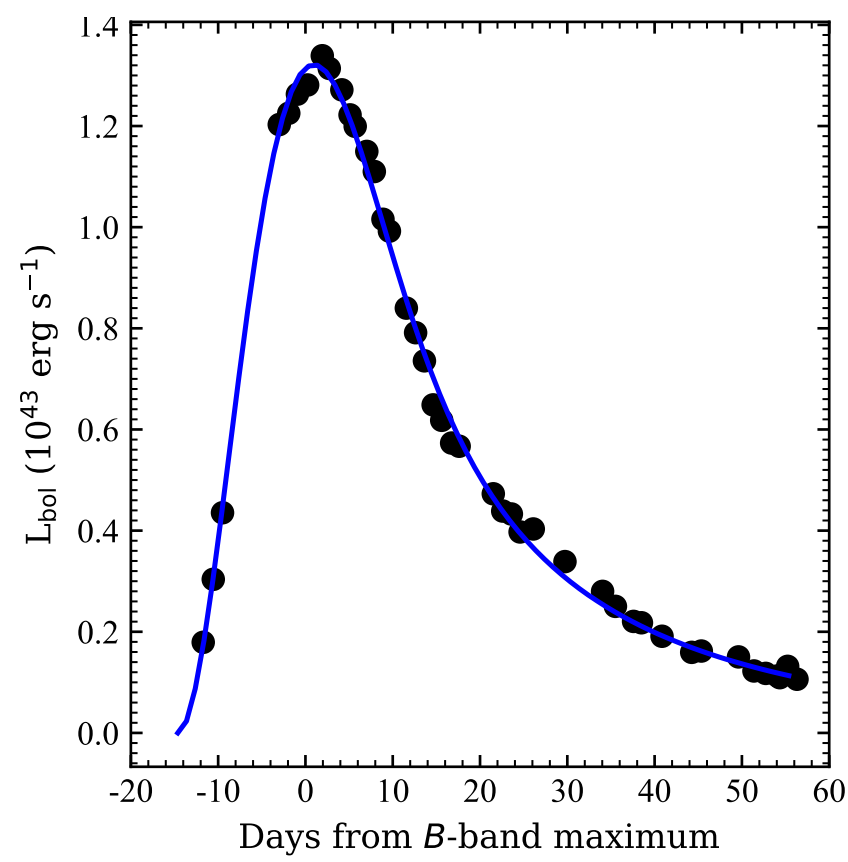

Figure 9. Quasi-bolometric light curve (dots) with an Arnett (1982) radiation diffusion model (blue curve).

\& Morozova 2016; Li et al. 2019). The first light time of $57941.4 \pm 0.4 \mathrm{~d}$ is used in the following analysis.

\section{OPTICAL SPECTRA}

\subsection{Temporal Evolution of the Spectra}

The optical spectral evolution of SN 2017fgc is displayed in Figure 10. The early-time spectra are characterized by prominent absorption lines of intermediate-mass elements (IMEs) and ionized iron-group elements (IGEs), including Ca II H\&K, Fe II $\lambda \lambda 4404,5018, \mathrm{Mg}$ II $\lambda 4481$, Si II $\lambda 5051$, Fe III $\lambda 5129$, S II $\lambda \lambda 54668,5654$, Si II $\lambda 6355$, and the Ca II NIR triplet. About two weeks before the $B$-band maximum, the absorption line near $4300 \AA$ could be due to blended Fe II $\lambda 4404$ and $\mathrm{Mg}$ II $\lambda 4481$, while the broad absorption near $4800 \AA$ could be a blend of Fe III $\lambda 5129$, Fe II $\lambda \lambda \lambda 4924$, 5018,5169 , and Si II $\lambda 5051$. The distinct absorption features near $3700 \AA$ and $7800 \AA$ can be identified as Ca II H\&K and the Ca II NIR triplet, respectively.

After about one week before maximum light, the "W"shaped S II absorption features near $5400 \AA$ and Si II $\lambda 5972$ near $5800 \AA$ begin to emerge in the spectra. The minimum of the Si II $\lambda 6355$ absorption line shifted redward gradually with the decreasing photospheric velocity, while the IGEs and sulfur gradually gain strength. At around the $B$-band maximum, the spectra are still dominated by the "W"-shaped S II absorption features and distinct absorption lines of Ca II H\&K, Si II $\lambda 6355$, and the Ca II NIR triplet. The blended absorption lines of Fe III $\lambda 5129$, Fe II $\lambda \lambda \lambda 4924,5018,5169$, and Si II $\lambda 5051$, as well as the blended absorption lines of 
Fe II $\lambda 4404$ and $\mathrm{Mg}$ II $\lambda 4481$, are also notable. At about ten days after maximum light, the "W"-shaped S II absorption line becomes very weak, while the features of $\mathrm{Si}$ II $\lambda 6355$ and the Ca II NIR triplet still remain prominent. At about one month after maximum, the Ca II NIR triplet and Ca II H\&K absorption lines are still the dominant spectral features. By the time when the SN enters the early nebular phase, features of IGEs start to emerge in the spectra.

The spectra of SN 2017fgc at four selected epochs are compared with those of several well-observed SNe Ia having similar $\Delta m_{15}(B)$, as illustrated in Figure 11. Panel (a) shows the comparison of the $t \approx-12 \mathrm{~d}$ spectra, including SNe 2017fgc (this paper), 2002bo, 2003du, 2005cf, 2006X, $2011 \mathrm{fe}$, and 2013gs. One can see that both SN $2017 \mathrm{fgc}$ and the comparison SNe Ia have strong absorption features due to $\mathrm{Ca}$ II $\mathrm{H} \& \mathrm{~K}$ and $\mathrm{Si}$ II $\lambda 6355$. The three HV objects (with pEW being $211.1 \pm 19.2 \AA$ for SN 2002bo, 211.6 $\pm 26.0 \AA$ for SN 2006X, and $188.7 \pm 15.6 \AA$ for SN 2017 fgc, respectively) are found to have systematically stronger $\sim 4800 \AA$ absorption than three NV ones (with pEW being $86.8 \pm 10.1 \AA$ for SN $2003 \mathrm{du}, 146.8 \pm 3.3 \AA$ for SN $2005 \mathrm{cf}$, and $122.2 \pm 4.0 \AA$ for $\mathrm{SN} 2011 \mathrm{fe}$, respectively) at similar phases. While the pEW is measured to be $142.5 \pm 24.4 \AA$ for SN 2013gs (HV), which is comparable to that measured for SN 2005cf (NV). Previous studies suggest that the absorption near $4800 \AA$ could be due to a blend of Fe III $\lambda 5129$, Fe II $\lambda \lambda \lambda 4924,5018$, 5169 , and Si II $\lambda 5051$ lines.

Figure 11(b) shows the comparison at $t \approx 1$ week before maximum light. The absorption-line strengths of IMEs are enhanced in the spectra of SN 2017fgc and the comparison SNe Ia. At this phase, the "W"-shaped S II absorption features near $5400 \AA$ start to emerge in spectra of all $\mathrm{SNe}$ Ia in our sample. However, we notice that the Fe II and $\mathrm{Mg}$ II blended feature near $4500 \AA$ is stronger in SNe 2002bo, 2006X, 2013gs, and $2017 \mathrm{fgc}$ relative to the three objects in the NV SN Ia subclass.

The spectra near maximum light are displayed in Figure 11(c). At this phase, the blended features near $4800 \AA$ of SN 2017fgc (with pEW being 227.9 $\pm 3.5 \AA$ ) are comparable to those of HV objects (with pEW being 204.2 $\pm 14.1 \AA$ for SN 2002bo, 213.4 $\pm 6.4 \AA$ for SN 2006X, and 156.6 $\pm 9.3 \AA$ for SN 2013gs), but they are obviously stronger than those of the NV counterparts (with pEW being $121.1 \pm 13.6 \AA$ for SN $2003 \mathrm{du}, 130.1 \pm 3.2 \AA$ for SN $2005 \mathrm{cf}$, and $125.1 \pm 3.2 \AA$ for $\mathrm{SN} 2011 \mathrm{fe})$. The similar situation is found for the blended features near $4300 \AA$, with pEW being $106.5 \pm 9.6 \AA$ for SN 2002bo, 102.2 $\pm 4.0 \AA$ for SN 2006X, 100.6 $\pm 6.1 \AA$ for SN 2013gs, $110.7 \pm 2.4 \AA$ for SN $2017 f g c$, and $86.4 \pm 10.0 \AA$

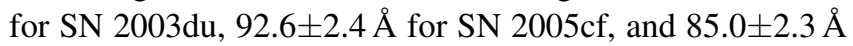
for SN 2011fe. The velocity measured for SN $2017 \mathrm{fgc}$ from the absorption-line minimum of $\mathrm{Si}$ II $\lambda 6355$ at maximum light is $15,000 \pm 150 \mathrm{~km} \mathrm{~s}^{-1}$, which is about $2000 \mathrm{~km} \mathrm{~s}^{-1}$

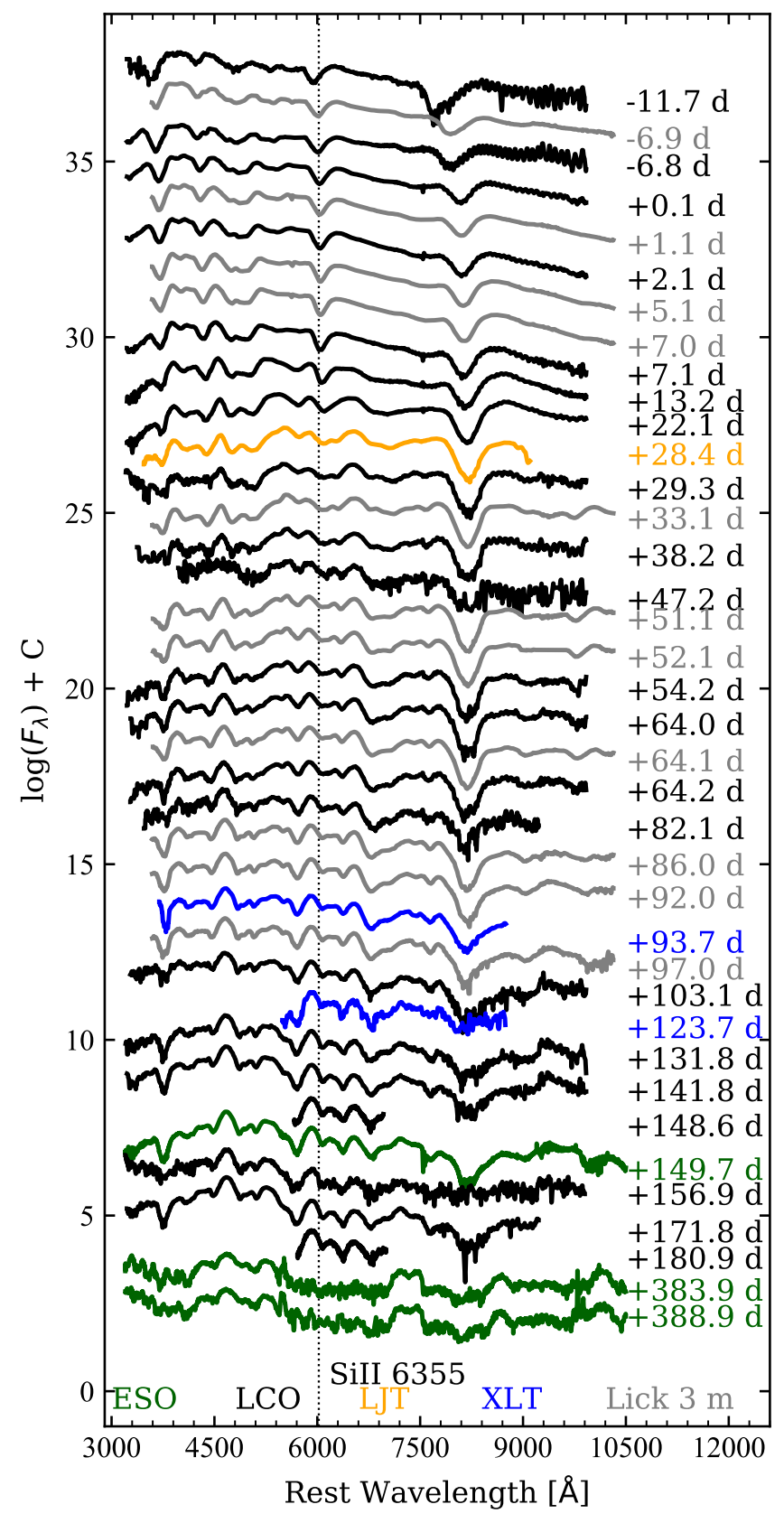

Figure 10. Optical spectral evolution of SN 2017fgc. All of the spectra have been corrected for the redshift of the host galaxy and reddening. The epochs shown on the right side represent the phases in days relative to $B$-band maximum light. The dashed line marks the center of the $\mathrm{Si}$ II $\lambda 6355$ line profile at $+0.04 \mathrm{~d}$ from $B$-band maximum. The colors of the spectra represent data from different instruments.

faster than that of $\mathrm{SN} 2002$ bo (i.e., $\sim 13,200 \mathrm{~km} \mathrm{~s}^{-1}$ ). $R$ (Si II), defined as the line-strength ratio of Si II $\lambda 5972$ to $\mathrm{Si}$ II $\lambda 6355$ (Nugent et al. 1995), is measured to be $0.04 \pm 0.01$ for SN $2017 \mathrm{fgc}$, noticeably smaller than that of the NV SN 2005cf ( $R$ (Si II $)=0.28 \pm 0.04$; Wang et al. 2009 b), perhaps suggesting a relatively higher photospheric 


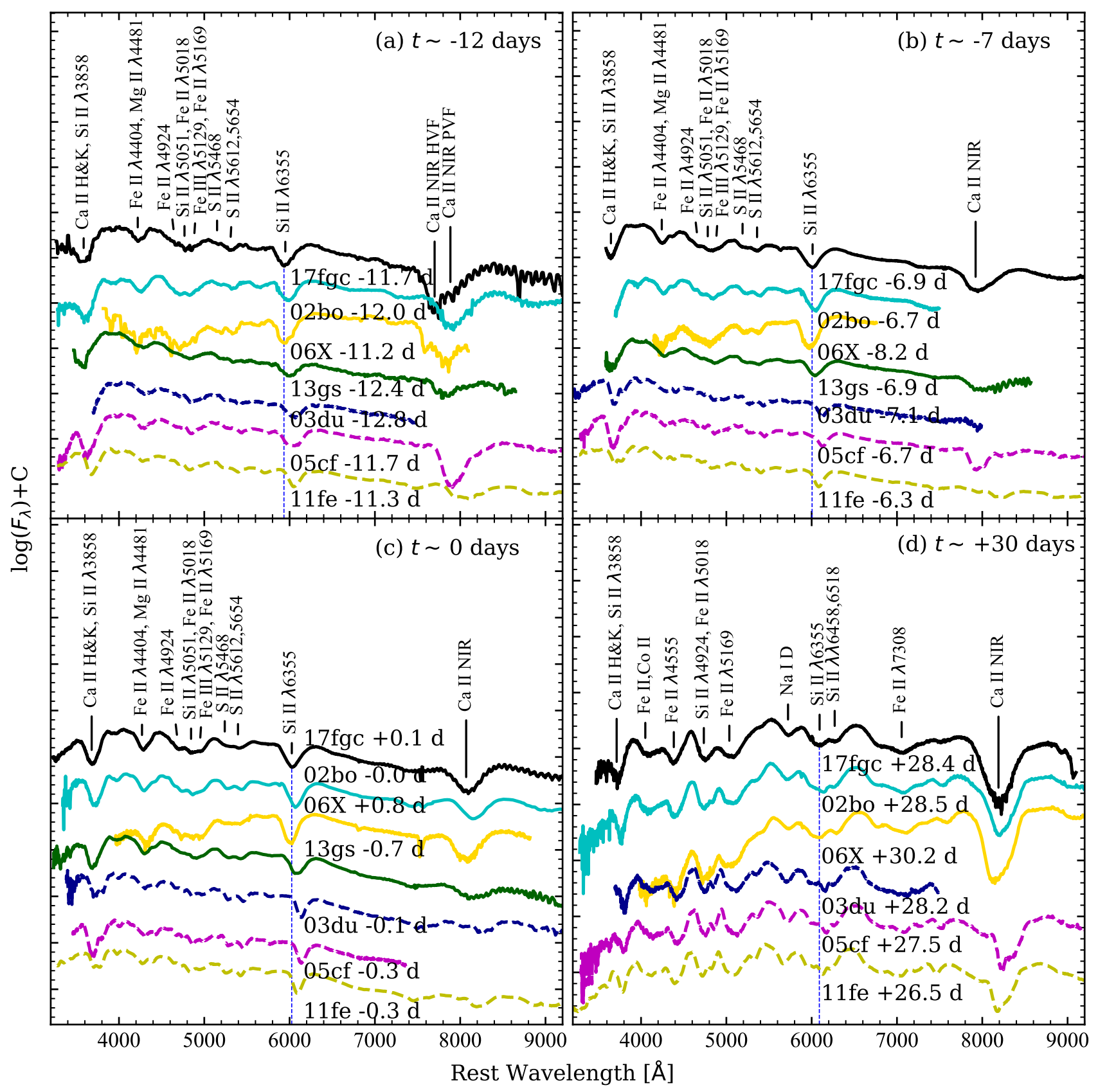

Figure 11. Spectra of SN $2017 \mathrm{fgc}$ at $t \approx-12,-7,+0$, and $+30 \mathrm{~d}$ relative to $B$-band maximum, compared with spectra of SNe 2002bo (Benetti et al. 2004), 2003du (Stanishev et al. 2007), 2005cf (Wang et al. 2009b), 2006X (Wang et al. 2008b), 2011fe (Zhang et al. 2016b), and 2013gs (Zhang et al. 2019) at comparable phases. The spectra of HV SNe Ia are represented with solid lines while those of the NV ones are denoted with dashed lines. All of the above spectra have been corrected for reddening and redshift of the host galaxy.

temperature of SN 2017fgc around maximum light. In Figure 12 , the line ratio $R(\mathrm{Si}$ II) of a few representative $\mathrm{SNe}$ Ia of different subclasses are plotted versus their $B$-band magnitude decline $\Delta m_{15}(B)$. As expected, the fast decliners tend to have larger $R(\mathrm{Si}$ II) ratio and vice versa. Compared to the NV SNe Ia with similar decline rates, the HV ones have systematically smaller values of $R(\mathrm{Si} \mathrm{II})$, which provides additional evidence for different properties between these two subclasses. A systematic lower $R(\mathrm{Si}$ II) ratio in- dicates that the HV SNe Ia might have higher photospheric temperature and experienced more complete burning or they suffered from interaction of ejecta with CSM or companion stars (Zhao et al. 2015, 2016).

Figure 11(d) shows the spectral evolution at $t \approx 30 \mathrm{~d}$ after maximum light. The absorption profiles of SN $2017 \mathrm{fgc}$ and the comaprison $\mathrm{SNe}$ Ia are well developed and tend to have uniform morphologies. Form the $t \sim 1$ month spectra, the pseudo-equivalent width ( $\mathrm{pEW}$ ) of $\mathrm{Ca}$ II NIR triplet of $\mathrm{SNe}$ 


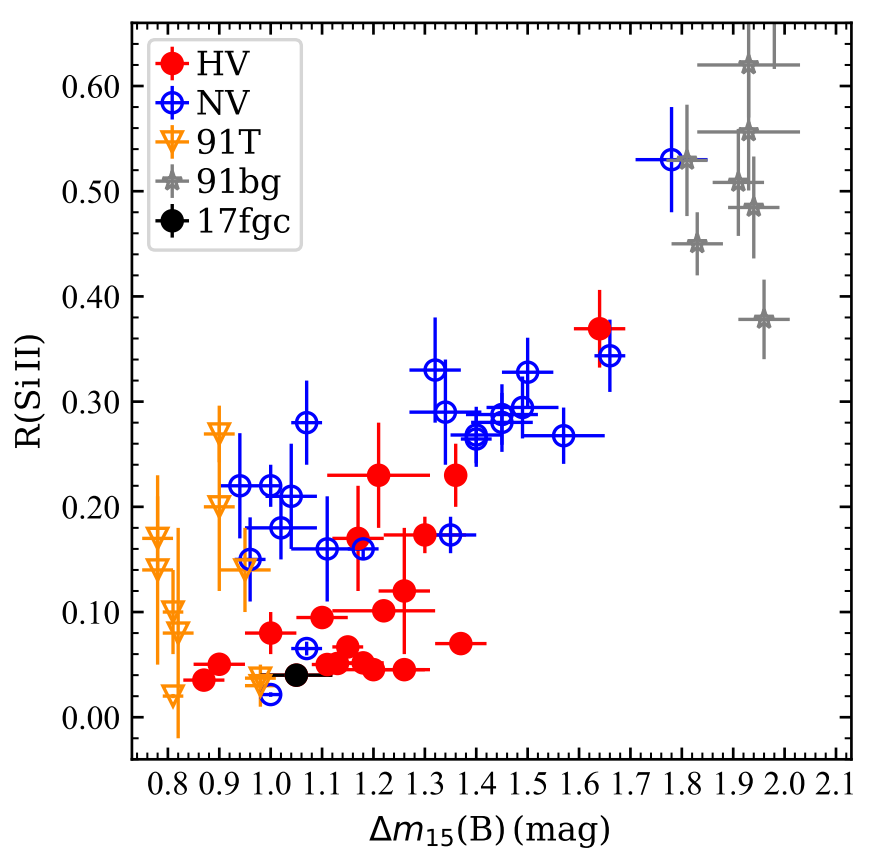

Figure 12. Line ratio $R(\mathrm{Si}$ II) of several well studied SNe Ia versus luminosity indicator $\Delta m_{15}(B)$. Some data are taken from Benetti et al. (2004) and Blondin et al. (2012), while others are taken from Stanishev et al. (2007) for SN 2003du, Wang et al. (2009b) for SN 2005cf, Zeng et al. (2021) for SN 2017hpa, Li et al. (2019) for SN 2018oh, Wang et al. (2008b) for SN 2006X, Zhang et al. (2010) for SN 2007gi, Zhang et al. (2019) for SN 2013gs, and Kawabata et al. (2020) for SN 2019ein, respectively. The $R(\mathrm{Si}$ II) ratios for $\mathrm{SNe}$ 1998bp, 1998es, 1999gp, 2001eh, and 2011fe are calculated using the code respext with the spectra near maximum light (Stahl et al. 2020b). The HV SNe Ia are represented by red dots while the $\mathrm{NV}$ ones are shown by blue open circles. The peculiar object $\mathrm{SNe}$ 1991bg and 1991T are plotted by red and blue dots, respectively, while SN $2017 \mathrm{fgc}$ is overplotted by black dot.

2002bo, 2006X, 2017fgc, 2005cf and 2011fe are measured as $479.0 \pm 17.5 \AA, 475.6 \pm 9.0 \AA, 492.2 \pm 13.2 \AA, 73.9 \pm 2.9 \AA$, $89.5 \pm 0.5 \AA$, respectively. We see that the Ca II NIR absorption lines in the spectra of HV SNe 2002bo, 2006X, and $2017 \mathrm{fgc}$ are much stronger than those of NV SNe 2005cf and 2011fe. By $t \approx 1$ month, the Fe II features gain strength and gradually dominate the wavelength region 4700-5000 $\AA$.

Nebular spectra at $t \approx 170 \mathrm{~d}$ and $t \approx 400 \mathrm{~d}$ after maximum light are shown in Figures 13(a) and 13(b), respectively. The spectra of SN $2017 \mathrm{fgc}$ are well developed at such late phases and characterized by the forbidden lines of singly and doubly ionized IGEs, such as the $[\mathrm{Fe} \mathrm{II}]$ and $[\mathrm{Fe} \mathrm{III}]$ features at $\sim$ 4700, 5000, 6500, and $\sim 7000 \AA$, as well as [Co II] and [Co III] at $\sim 5800$ and $\sim 6500 \AA$. The same lines are commonly seen in the comparison SNe Ia at similar phases. We notice that the emission profile of $[\mathrm{Fe}$ II $] /[\mathrm{Fe}$ III $]$ at $\sim 5000 \AA$ is stronger in the NV SNe Ia (i.e., with the pEW being measured as $63.8 \pm 2.3 \AA$ and $62.8 \pm 1.6 \AA$ for SN 2003du and SN $2011 \mathrm{fe}$, respectively) than in HV ones (i.e., with the pEW being measured as $24.8 \pm 7.3 \AA$ and $26.0 \pm 1.9 \AA$ for $\mathrm{SN}$ 2017 fgc and SN 2006X, respectively). From the $t \approx+384 \mathrm{~d}$ spectrum, we measured the velocity shift of [Fe II] $\lambda 7155$ and [Ni II] $\lambda 7378$ as $+2220 \pm 260 \mathrm{~km} \mathrm{~s}^{-1}$, and that measured from the $t \approx+389 \mathrm{~d}$ spectrum is $+1640 \pm 580 \mathrm{~km} \mathrm{~s}^{-1}$; these are consistent with the trend that HV SNe Ia tend to have redshifted Fe II/Ni II velocity in the nebular phase (Maeda et al. 2010; Maguire et al. 2018).

\subsection{Ejecta Velocity}

The methods described by Zhao et al. $(2015,2016)$ were utilized to measure the ejecta velocity from the absorption lines, such as S II $\lambda \lambda 5460,5640$, Si II $\lambda 5972$, Si II $\lambda 6355$, and the Ca II NIR triplet, are shown in Figure 14. The photospheric velocity measured from Si II $\lambda 6355$ at $t \approx-11.7 \mathrm{~d}$ is $\sim 18,900 \mathrm{~km} \mathrm{~s}^{-1}$, comparable to that of the Si II $\lambda 5972$ $\left(\sim 18,000 \mathrm{~km} \mathrm{~s}^{-1}\right)$ and S II lines $\left(\sim 19,000 \mathrm{~km} \mathrm{~s}^{-1}\right)$, but much slower than the high-velocity-feature (HVF) velocity inferred from the Ca II NIR triplet $\left(\sim 32,000 \mathrm{~km} \mathrm{~s}^{-1}\right)$. At the time of $B$-band maximum, the velocity of Si II $\lambda 6355$ is measured as $15,000 \pm 150 \mathrm{~km} \mathrm{~s}^{-1}$, significantly larger than the typical value (i.e, $\sim 11,800 \mathrm{~km} \mathrm{~s}^{-1}$ ) of normal velocity (NV) SNe Ia. We thus put SN 2017 fgc into the HV subclass according to the classification criteria proposed by Wang et al. (2009a).

Applying a linear fitting to the velocities of Si II $\lambda 6355$ measured during the period $t \approx 0 \mathrm{~d}$ to $t \approx+10 \mathrm{~d}$, we derive the velocity gradient as $120 \pm 10 \mathrm{~km} \mathrm{~s}^{-1} \mathrm{~d}^{-1}$, suggesting that SN 2017fgc belongs to the HVG subclass. Figure 14 shows the velocity evolution of some IMEs, including S II, Si II, and Ca II. The velocities of Si II $\lambda 5972$ and the Ca II NIR triplet photospheric component show similar evolution, while Si II $\lambda 6355$ exhibits slightly higher velocities. The HVF of the Ca II NIR triplet has the highest velocity. The photospheric velocity evolution of SN $2017 \mathrm{fgc}$, as derived from the minimum of Si II $\lambda 6355$ absorption, is shown in Figure 15, together with that of the comparison SNe Ia, including SNe 2002bo, 2005cf, 2006X, and 2011fe. As can be seen, SN $2017 \mathrm{fgc}$ exhibits a velocity evolution similar to that of SN 2006X. The basic photometric and spectroscopic parameters of SN $2017 \mathrm{fgc}$ are listed in Table 5.

\section{DISCUSSION}

\subsection{Velocity and Velocity Gradient}

The large velocity $\left(\sim 15,000 \pm 150 \mathrm{~km} \mathrm{~s}^{-1}\right)$ and velocity gradient $\left(\sim 120 \pm 10 \mathrm{~km} \mathrm{~s}^{-1} \mathrm{~d}^{-1}\right)$ clearly put $\mathrm{SN}$ $2017 \mathrm{fgc}$ into the subclasses of both HV and HVG. In general, most HV SNe Ia belong to the HVG subclasses, while the NV SNe Ia correspond to the LVG ones (Wang et al. 2009a; Silverman et al. 2012b), as shown in Figure 16(a). However, there are also outliers such as SN 2017hpa, which has a normal velocity around maximum light $\left(\sim 9550 \mathrm{~km} \mathrm{~s}^{-1}\right)$ 


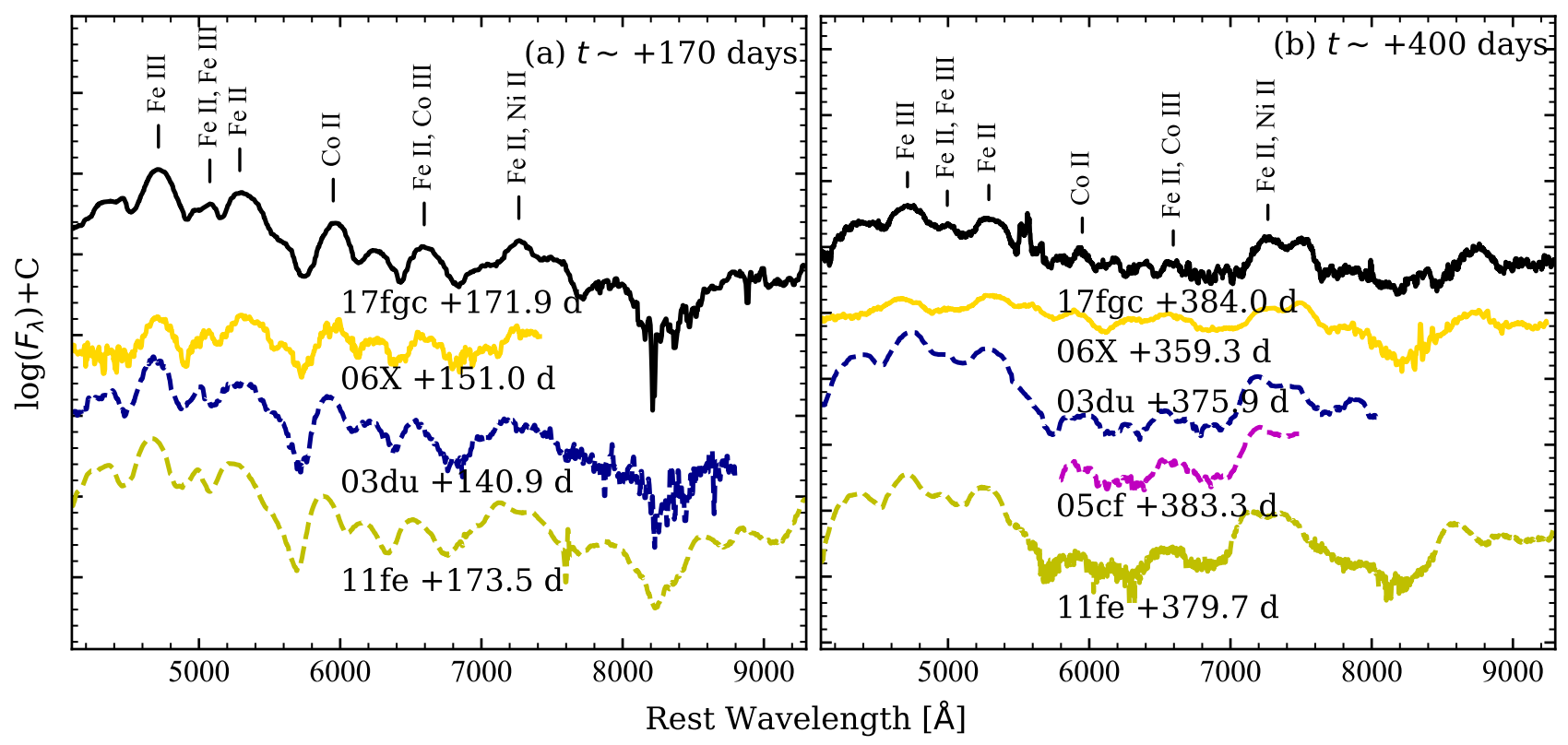

Figure 13. Nebular-phase spectra of SN $201 \mathrm{fgc}$ at $t \approx+170 \mathrm{~d}$ and $+384 \mathrm{~d}$ relative to $B$-band maximum, compared with spectra of SNe $2003 \mathrm{du}$ (Stanishev et al. 2007), 2005cf (Wang et al. 2009b), 2006X (Wang et al. 2008b), 2011fe (Zhang et al. 2016b), and 2013gs (Zhang et al. 2019) at comparable phases. The HV SNe Ia are represented with solid lines while the NV ones are dashed lines. All of the spectra have been corrected for reddening and redshift of the host galaxy.

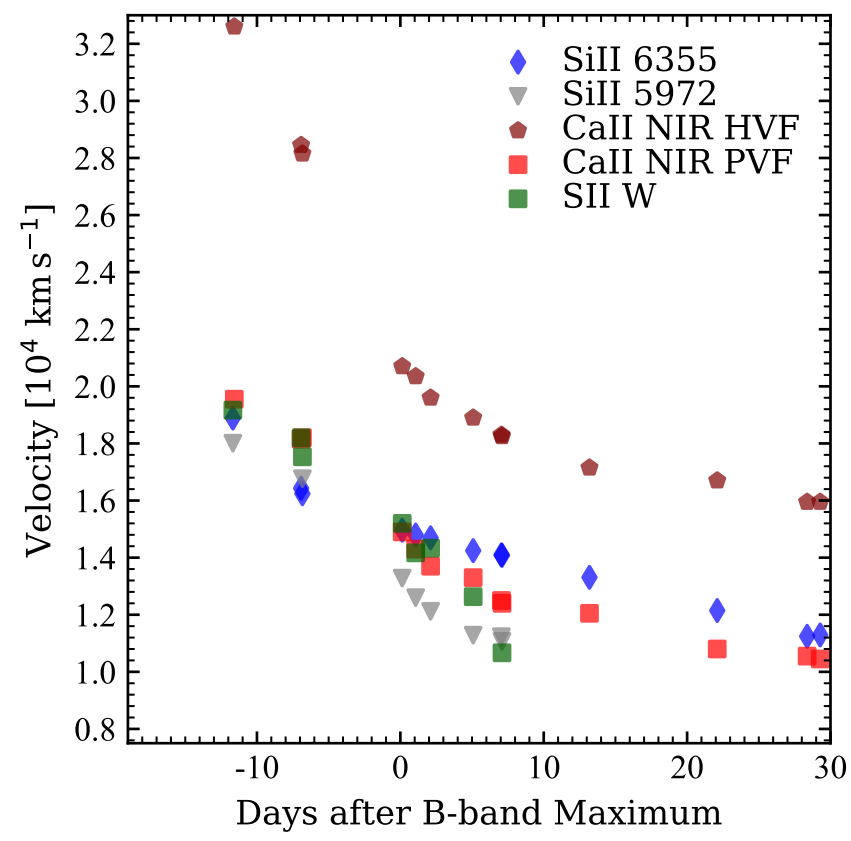

Figure 14. Velocity evolution of different elements measured from spectra of SN $2017 \mathrm{fgc}$. Note that for Ca II, both the detached highvelocity feature (HVF) and photospheric-velocity feature (PVF) are shown for comparison.

but a large velocity gradient $\left(\sim 130 \mathrm{~km} \mathrm{~s}^{-1}\right)$ (Zeng et al. 2021). This indicates that HVG SNe Ia could have multiple physical origins. This is also demonstrated by the correlation between velocity gradient and $\Delta m_{15}(B)$ (Phillips 1993), as shown in Figure 16(b), where no obvious correla- tion exists between velocity gradient and the luminosity indicator $\Delta m_{15}(B)$ while only the LVG sample possibly shows a weak correlation between these two observables.

Previous studies have shown that the difference in velocity gradient may be related to the different nature of the explosions or the mixture of SN Ia ejecta (e.g., Benetti et al. 2004; Sahu et al. 2013). Different viewing angles in an off-center ignition during the explosion of SNe Ia will lead to variations in the observed velocity gradient (Maeda et al. 2010). However, this explanation has difficulty explaining the observed fact that HVG (HV) SNe Ia preferentially occur in the inner region of the galaxy (Wang et al. 2013). Woosley et al. (2009) and Blondin et al. (2012) found that varying the criterion of the deflagration-to-detonation transition (DDT) will cause varying velocity gradients in a wide range. The HVG subclass may result from adequate mixing of heavy elements in the SN ejecta, while the counterparts with low velocity gradient may suffer less mixing in the ejecta (Blondin et al. 2012; Sahu et al. 2013). As suggested by Zeng et al. (2021), the large velocity gradient seen in SN 2017hpa could be caused by the effective mixing of heavy elements in the SN ejecta.

\subsection{Light-Curve Features}

During their study of the typical HV object SN 2006X, Wang et al. (2008a) noticed that it exhibited relatively flat tail evolution starting around $+40 \mathrm{~d}$ after maximum light. A recent statistical study based on a large sample indicates that the excess blue flux is common for the HV group of $\mathrm{SNe}$ Ia (Wang et al. 2019b; Li et al. 2021). For SN 2017fgc, the de- 


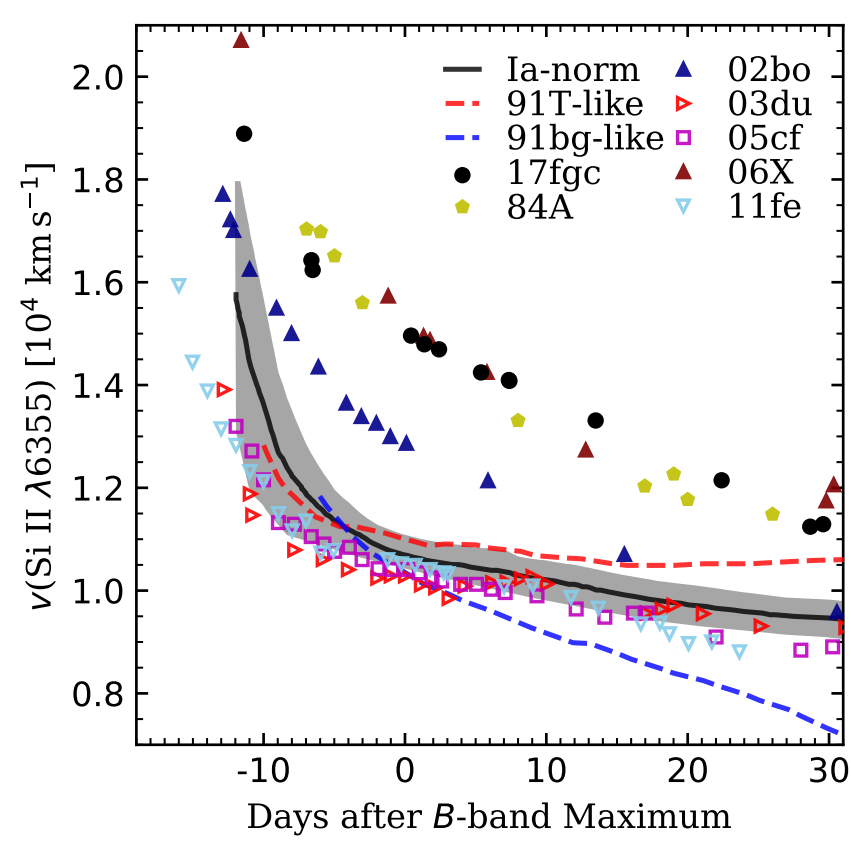

Figure 15. Evolution of the expansion velocity of SN $2017 \mathrm{fgc}$ as derived from the minimum of the Si II $\lambda 6355$ absorption line, compared with those of HV sample of SNe Ia, including 1984A, 2002bo, 2006X, and NV sample including SN 2003du, SN 2005cf, SN 2011 fe. The average velocity curves obtained for SN 1991T-like and SN 1991bg-like SNe are overplotted with red and blue dashed lines, respectively. The normal subclass of SNe Ia is plotted with a black solid line. The shaded region represents the $1 \sigma$ uncertainty for the mean velocity curve of NV SNe Ia. The values of SN 1984A are taken from Barbon et al. (1989), SN 2002bo from Benetti et al. (2004), SN 2006X from Wang et al. (2008b), SN 2005cf from Wang et al. (2009b), and SN 2011 fe from Zhang et al. (2016b). The region of NV SNe Ia is extracted from Wang et al. (2009a).

cline from peak brightness measured at $t \approx 60 \mathrm{~d}$ is $2.96 \mathrm{mag}$ in $B$ and 2.34 mag in $V$. The corresponding values are much smaller than in NV SNe Ia with similar $\Delta m_{15}(B)$, but are consistent with the behavior of HV SNe Ia. Figure 17 shows the results measured for SN 2017fgc and the comparison sample from Wang et al. (2019b). For the NV sample of SNe Ia, more-luminous objects tend to have brighter tails and slower decline rates, while this tendency shows large scatter in the $B$ band owing to the abnormally bright tails exhibited by the HV subsamples (Wang et al. 2019b). This is primarily due to the fact that HV SNe Ia tend to show excess emission in the early nebular phase, which can be caused by light scattering by the surrounding CSM (Wang et al. 2019b). This scenario is favored by the detections of nearby light echoes around SN 2006X (Wang et al. 2008a) and SN 2014J (Yang et al. 2018).

Besides the flatter bluer-band light-curve evolution in the early nebular phase, HV SNe Ia seem to share another lightcurve feature: a stronger secondary shoulder or maximum in the $R / r$ and $I / i$ bands. This seems to apply for SN 2017 fgc. Following Stahl et al. (2020b), the Gaussian-process lightcurve fitting implemented in SNooPy2 is employed to obtain the secondary peak magnitudes of SN Ia light curves in the $R / r$ and $I / i$ bands. The resulting absolute $\operatorname{Rr} I i$-band secondary peak magnitudes of $92 \mathrm{SNe}$ Ia (including $26 \mathrm{HV}$ and $66 \mathrm{NV}$ SNe Ia) are plotted as a function of Si II velocity in Figure 18. The black line with square symbols represent the binned average (i.e., a bin of $1,000 \mathrm{~km} \mathrm{~s}^{-1}$ is used to calculate the average value). The pearson coefficient is estimated as 0.94 and -0.88 for the binned $r$-band and $i$-band data, respectively. The corresponding p-values are 0.02 and 0.04 , respectively, suggesting that the shoulder/secondary peak features could be correlated with the Si II velocity at a confidence level of $\sim 2 \sigma$.

The NIR light curves of SNe Ia have been occasionally studied theoretically. Several delayed-detonation models have been applied by Höflich (1995) to reconstruct the $I$-band and NIR light curves. They described the doublepeaked behavior as a temperature effect, in which the drop in temperature of the ejecta is compensated by the expansion of the photosphere. Pinto \& Eastman (2000) explained the secondary maximum in the NIR bands as the release of trapped radiative energy owing to the decrease in the flux mean opacity. Kasen (2006) found that the NIR secondary maximum of SNe Ia could be due to the ionization transition of IGEs in the ejecta from doubly to singly ionized, which in turn leads to a weakening of Fe III and Co III lines and a strengthening of Fe II and Co II lines (see also Jack et al. 2012; Dessart et al. 2014; Blondin et al. 2015). They suggested that the properties of the secondary maximum are related to $\Delta m_{15}(B)$ (and hence the peak luminosity), being more prominent in the brighter SNe Ia. Moreover, a small fraction of stable IGEs may be produced depending on the metallicity of the progenitor WD during the burning to nuclear statistical equilibrium (Kasen 2006; Kasen \& Woosley 2007). The peak magnitude of the secondary maximum increases with the growing of the stable iron core, while the increase in metallicity of the progenitor increases the size of the iron core. In comparison NV SNe Ia, the more pronounced shoulder/secondary peak features of HV SNe Ia seen in $R / r$ and $I / i$ bands might be related to that the latter subclass have more metal-rich progenitor populations (Wang et al. 2013; Pan 2020).

\subsection{Absorption of Intermediate-Mass and Iron-Group Elements}

It is common that HV SNe Ia also show stronger absorption of Si II $\lambda 6355$ as evidenced by the fact that this subclass overlaps greatly with the BL subclass. According to Kasen (2006), the SN ejecta becomes very effective in redistributing the blue/UV photons to longer wavelengths when the iron-rich layers of the SN ejecta cools down to $\sim 7000$ 


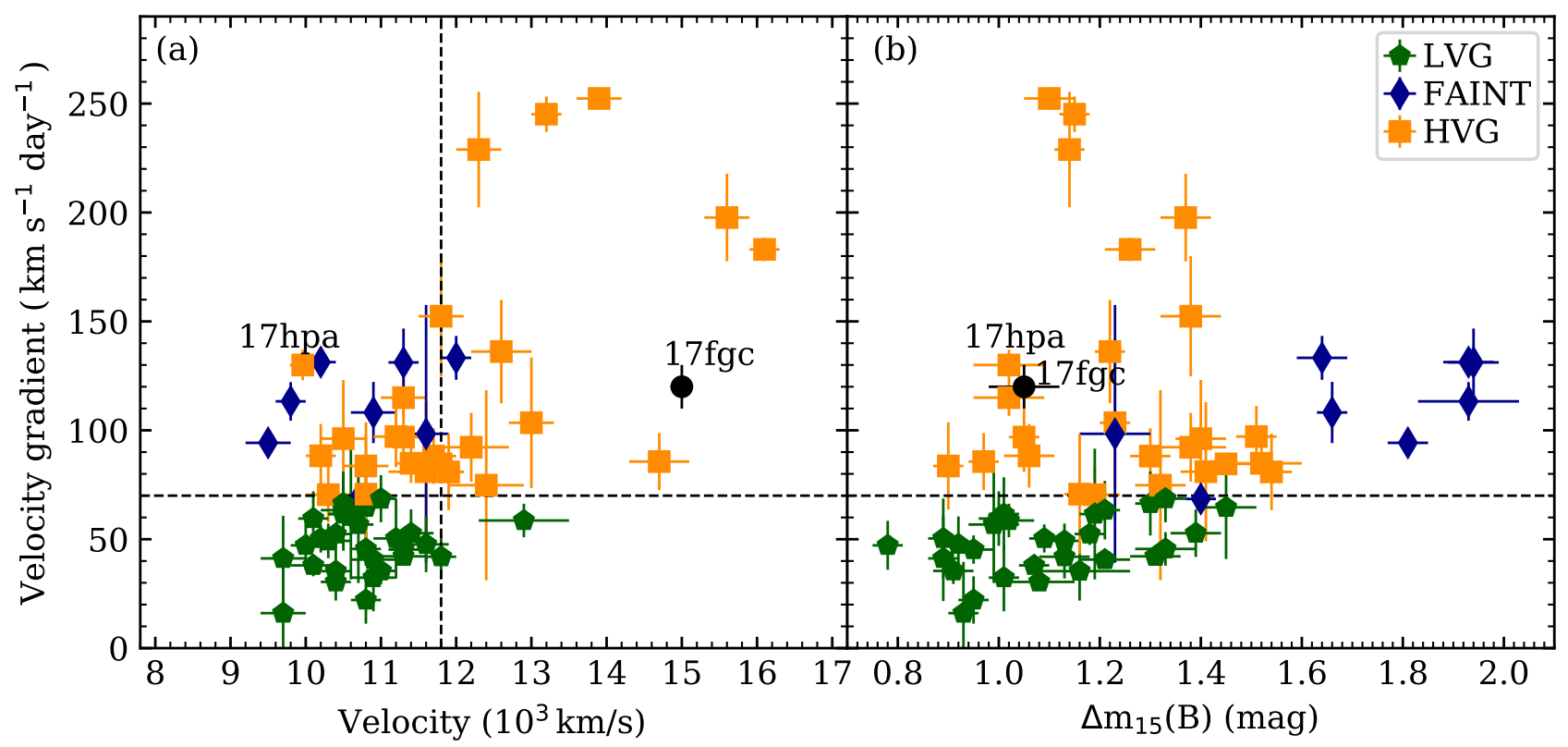

Figure 16. The spectroscopic subclassification of SN 2017fgc (as marked with a black dot) based on the scheme of Benetti et al. (2005). (a) The scatter plot of the Si II velocity versus the velocity gradient. The velocity gradients of SN 2005cf and SN 2018oh are taken from Wang et al. (2009b) and Li et al. (2019), respectively, and those of the other objects are from Benetti et al. (2005) and Folatelli et al. (2013). The velocities are taken from Silverman et al. (2012b) and Wang et al. (2019b). The horizontal dashed line in the right pannel marks the boundary between HVG and LVG, which is $70 \mathrm{~km} \mathrm{~s}^{-1} \mathrm{~d}^{-1}$ as defined by Benetti et al. (2005), while the vertical dashed line represents the boundary between the HV and NV SNe Ia as defined by Wang et al. (2009b). (b) $\Delta m_{15}(B)$ is plotted versus the velocity gradient measured from the Si II $\lambda 6355$ absorption line in the near-maximum-light spectra.

Kelvin, which leads to the rebrightening of the NIR light curves. Jack et al. (2012) also found that the recombination of Fe III to Fe II is responsible for the second maximum features in NIR bands. Silverman et al. (2012a) suggested that the pEW of Mg II complex and Fe II complex are correlated to the SALT2 colour, with the HV SNe being redder and having larger pEWs (see also Nordin et al. 2011; Walker et al. 2011). To further investigate the discrepancy between HV and NV SNe Ia (including $66 \mathrm{NV}$ and $26 \mathrm{HV}$ ones), we examine the pEW of the blended absorption near $4400 \AA$ (including Fe II $\lambda 4404$ and Mg II $\lambda 4481$ ) and that near $4900 \AA ̊$ Aincluding Fe III $\lambda 5129$, Fe II $\lambda \lambda \lambda 4924,5018,5169$, and Si II $\lambda 5051)$, respectively dubbed as $\mathrm{pEW}(\mathrm{Mg}$ II) and $\mathrm{pEW}(\mathrm{Fe}$ II). The samples are the same as those used by Wang et al. (2019b). The code respext ${ }^{6}$ (Stahl et al. 2020b) is employed to estimate the pEWs of Fe II $\lambda 4404 / \mathrm{Mg}$ II $\lambda 4481$ and $\mathrm{Fe} \mathrm{II} / \mathrm{Fe}$ III blends from the spectra around maximum light, and linear fitting is utilized to infer the pEWs at $B$ band maximum.

Figure 19 (a) and (b) show the correlations between $\mathrm{pEW}(\mathrm{Mg}$ II $) / \mathrm{pEW}\left(\mathrm{Fe}\right.$ II) and $\Delta m_{15}(B)$. One can see that HV SNe Ia have on average larger $\mathrm{pEW}(\mathrm{Mg}$ II) and $\mathrm{pEW}(\mathrm{Fe}$ II) relative to NV SNe Ia. The p-values from the T

\footnotetext{
${ }^{6}$ https://github.com/benstahl92/respext
}

test of the pEW measured for HV and NV SNe Ia are 0.07 and 0.09 , corresponding to a significance of $\sim 2 \sigma$. This indicates that $\mathrm{HV}$ and NV SNe Ia may have different ejecta properties that might be related to explosion mechanism or progenitor system. Inspection on panels (c) and (d) of Figure 19 reveals that both $\mathrm{pEW}(\mathrm{Fe}$ II) and $\mathrm{pEW}(\mathrm{Mg}$ II) have a positive correlation with the Si II velocity. The difference in absorption features of IMEs and IGEs suggests that the outer ejecta of the HV SNe Ia may have experienced more complete burning than the NV subclass. This seems to be consistent with the delayed-detonation model, which involves an initial subsonically propagating mode of nuclear burning followed by a supersonically moving detonation front with some time delay (Khokhlov 1989). Several three-dimensional models based on delayed detonation Chandrasekhar-mass explosions also have been proposed in variants of DDT models (Khokhlov 2005; Bravo \& García-Senz 2008; Röpke \& Niemeyer 2007; Röpke et al. 2012). The reduced burning densities resulting from energy release in the subsonical propagation mode of nuclear burning leads to extensive burning of the remaining fuel (Seitenzahl et al. 2013). In addition, larger off-center distances of DDT models might lead to more silicon at high velocities (Höflich et al. 2002, 2006). For a given $\Delta m_{15}(B)$, the asymmetric detonation can aspherically push $\mathrm{Si}$ outward and make the Si II features form in the regions at higher ve- 

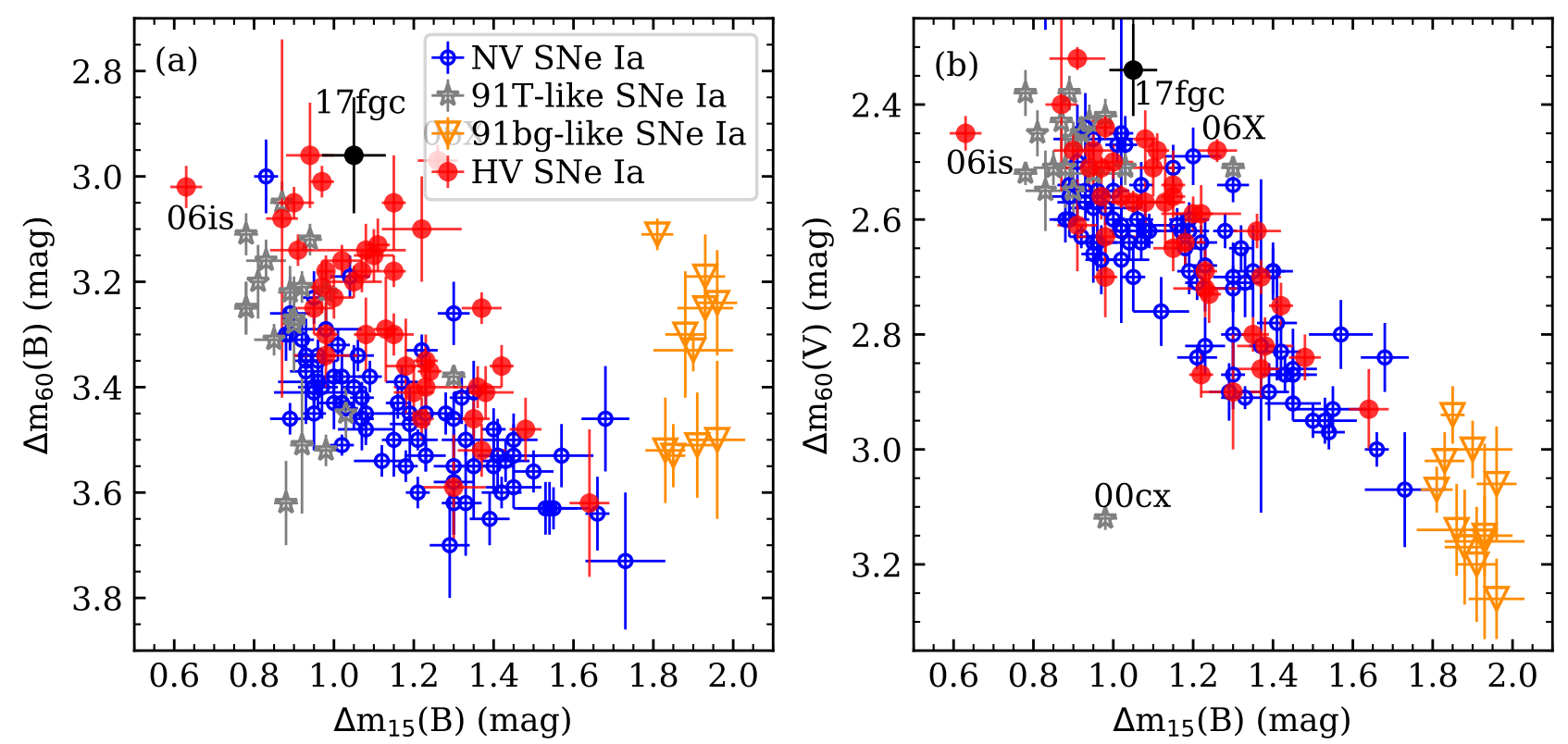

Figure 17. Tail brightness of SNe Ia, measured as the magnitude decline at $t \approx 60 \mathrm{~d}$ from the peaks of the (a) $B$ and (b) $V$ light curves, versus the luminosity indicator $\Delta m_{15}(B)$ that is measured as the magnitude decline within the first $15 \mathrm{~d}$ after $B$-band maximum (Phillips 1993). The data are taken from Wang et al. (2019b), while SN $2017 \mathrm{fgc}$ is overplotted as a black dot.

locities (Höflich et al. 2006; Wang et al. 2007; Yang et al. 2018; Cikota et al. 2019).

As an alternative, the larger pEWs seen in HV objects might be related to their environment metallicity, which could play a role in affecting the observed properties of SNe Ia (Domínguez et al. 2001; Timmes et al. 2003; Silverman et al. 2012b). Higher metallicity for HV SNe Ia is favored by the study of their birthplace environments (Wang et al. 2013; Pan 2020). According to Wang et al. (2013), HV SNe Ia likely have younger massive and metal-rich progenitor system than NV ones. The increase in stellar metallicity of progenitors could partially cause the higher Si II velocity seen in HV SNe Ia. The metal-rich stars could produce stronger outflows and produce more abundant CSM than the metal-pool counterparts (Wang et al. 2013), which is consistent with the observations that those showing blueshifted $\mathrm{Na}$ ID absorptions are more likely to be HV SNe Ia (Sternberg et al. 2011b; Foley et al. 2012; Wang et al. 2019b). The strong absorption lines of Fe II and IMEs seen in the spectra of SN 2017 fgc could thus originate from the metal-rich progenitor system, which will be further addressed below.

\subsection{Explosion Environment and its Metallicity}

In order to study the properties of the host galaxy NGC 474 , a total of 13 bands of photometric data ranging from the UV to the IR (including the NUV band from GALEX, five broad bands from SDSS, three NIR bands from 2MASS, and four NIR and mid-IR bands from Spitzer and WISE) from the
NASA/IPAC extragalactic database $\left(\mathrm{NED}^{7}\right)$ have been downloaded to construct its spectral energy distribution (SED). The stellar population synthesis code BC03 (Bruzual \& Charlot 2003) was employed to construct the spectral models with the adopted parameter configurations, including the initial mass function (IMF; Chabrier 2003) and the Padova 1994 evolutionary tracks and delayed-exponential star-formation history. The adopted SED-ftting method is described in Wei et al. (2021) while a $\chi^{2}$ minimization is used to fit the total stellar mass of NGC 474 (see also Lin et al. 2013). The observed SEDs and the best-fit templates of NGC 474 are shown in Figure 20. The logarithmic stellar mass of NGC 474 is estimated to be $10.72 \pm 0.02$ by fitting the observed SED with the stellar population synthesis model. Utilizing the Spitzer observations at $3.6 \mu \mathrm{m}$, Alabi et al. (2020) derived a similar logarithmic stellar mass of NGC 474 as $\sim 10.6$.

According to $\mathrm{Wu} \&$ Boada (2019), the relation between host galaxy stellar mass and metallicity can be characterized empirically as

$$
Z=-1.492+1.847 \log \left(M_{*} / M_{\odot}\right)-0.08026\left[\log \left(M_{*} / M_{\odot}\right]^{2},\right.
$$

where $Z$ is the oxygen abundance $12+\log (\mathrm{O} / \mathrm{H})$. Using this relation, a supersolar oxygen abundance of $9.08 \pm 0.12$ can be derived for NGC 474. From Figure 21, one can see that SN 2017fgc is consistent with the finding that HV SNe Ia tend to occur in more massive and metal-rich host galaxies. Also, the spectral survey for NGC 474 by Alabi et al. (2020)

\footnotetext{
${ }^{7}$ https://ned.ipac.caltech.edu/
} 


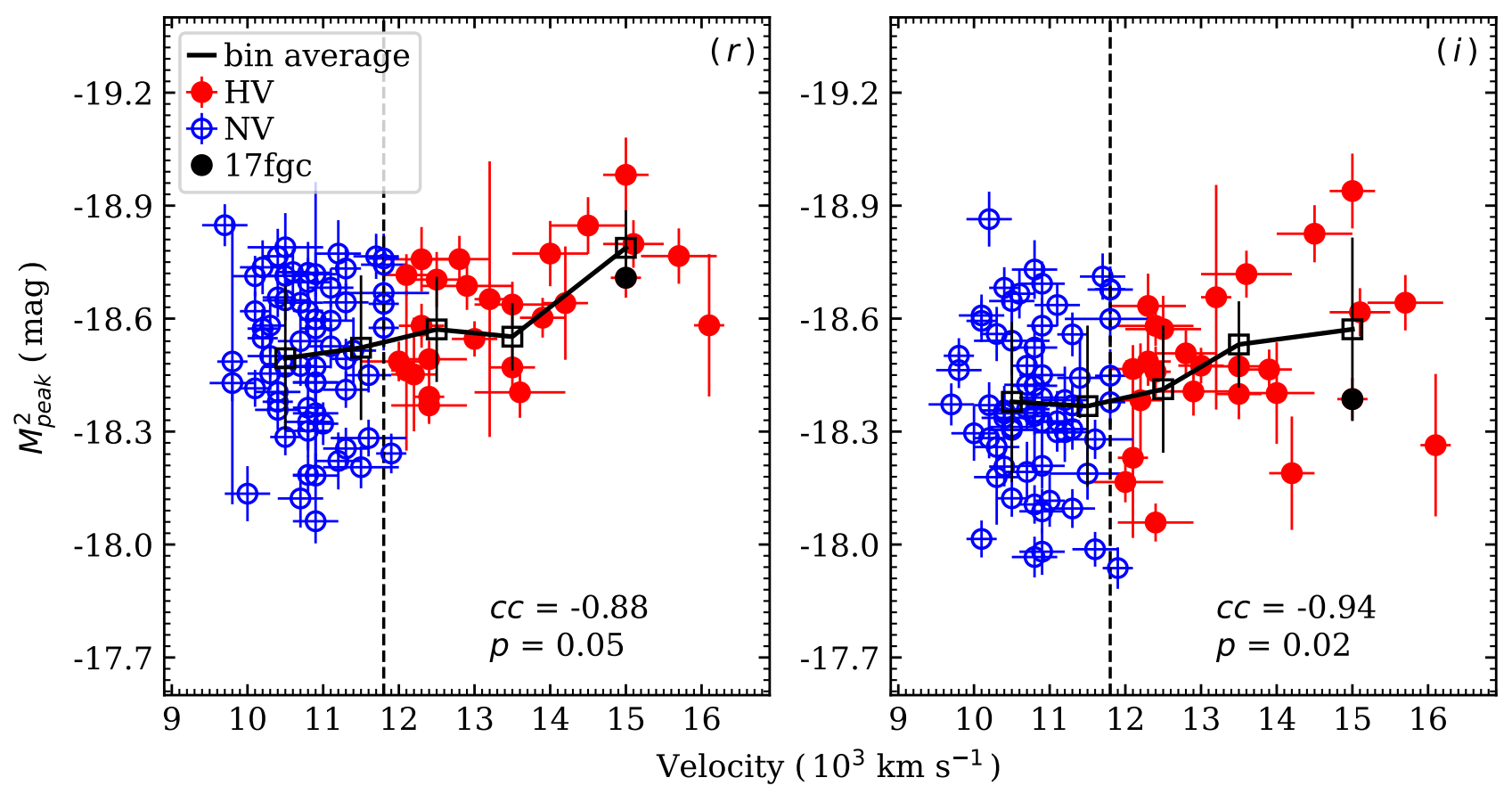

Figure 18. Left: The absolute secondary peak magnitude in $r$ band is plotted as a function of the Si II velocity of SNe Ia. The velocities are taken from Wang et al. (2019b). The black open square with black line represents the binned average, and $c c$ and $p$ are the correlation coefficient and p-value from pearson statistics (with significance level $\sim \mathbf{2} \sigma$ ), respectively. Right: Same, but in the $i$ band. The HV SNe Ia are represented with red dots while the NV ones are in blue open circles. The vertical dashed lines represent the boundary between the HV and NV SNe Ia as defined by Wang et al. (2009b).

shows that this galaxy has a slightly more metal-rich outer shell compared to the galaxy center. Recently, the detailed study by Fensch et al. (2020) showed that NGC 474 merged with a young metal-rich galaxy during its evolution.

Although a higher metallicity may help explain the observed properties seen in SN $2017 \mathrm{fgc}$ and some HV SNe Ia, we notice that SN 2017 fgc is located far away $(\sim 18.90 \pm$ $0.01 \mathrm{kpc}$ ) from the center of its host galaxy. Wang et al. (2013) initially found that HV SNe Ia tend to occur in more massive galaxy and explode near the center of the host galaxy, which is later confirmed by the Palomar Transient Factory (PTF) and the Berkeley SN Ia program sample (Pan et al. 2015; Pan 2020). It seems that SN 2017fgc is an outlier and does not follow this trend of HV SNe Ia. However, closer inspection of the host galaxy NGC 474 reveals that it is a massive lenticular galaxy that experienced a merger $\sim 2 \mathrm{Gyr}$ ago (Alabi et al. 2020; Fensch et al. 2020). SN 2017fgc is located in a gas bridge (see the left panel of Fig. 1) connecting the gas shell and the massive galaxy. We speculate that SN 2017 fgc could be ejected from the inner part of the companion galaxy NGC 470 during the merger took place at $~ 2 \mathrm{Gyr}$ ago, or formed as a result of some cold gas remained in the companion disk.

We attempted to measure the metallicity of the explosion site from both the multicolor photometry and MUSE IFU (Laurent et al. 2010) spectra but failed because no spectra near SN 2017fgc are available. According to Alabi et al. (2020), the centre of galaxy NGC 474 is dominated by slightly lower mass-weighted metallicity $[\mathrm{Z} / \mathrm{H}]=$ $0.14 \pm 0.08 \mathrm{dex}$ (corresponding to 0.72 times of the solar metallicity) while the outside shell has a solar metallicity $[\mathrm{Z} / \mathrm{H}]=-0.03 \pm 0.09$ dex (corresponding to 0.93 times of the solar metallicity). Thus, it is very likely that SN $2017 \mathrm{fgc}$ has a metal-rich progenitor system. Higher-metallicity WDs could produce relatively more stable, and less radioactive nucleosynthetic products owing to the overabundance of neutrons (Timmes et al. 2003), which is consistent with the observed evidence that relatively small amount of ${ }^{56} \mathrm{Ni}$ are found to be synthesized in the explosion of some $\mathrm{HV} \mathrm{SNe}$ Ia (Polin et al. 2019; Li et al. 2021). Moreover, a highermetallicity companion will produce more-abundant CSM, consistent with recent studies (Wang et al. 2013; Pan 2020; Li et al. 2021).

\section{CONCLUSION}

In this paper, we present extensive optical photometric and spectroscopic observations of the fast-expanding Type Ia SN $2017 \mathrm{fgc}$. This object can be put into the categories of both HVG and HV SNe Ia according to the classification schemes proposed by Benetti et al. (2005) and Wang et al. (2009a). It has a post-peak decline rate $\Delta m_{15}(B)=1.05 \pm 0.07 \mathrm{mag}$ and an absolute $B$-band magnitude $M_{\max }(B)=-19.32 \pm$ $0.13 \mathrm{mag}$. Using the radioactive-decay-driven radiation dif- 

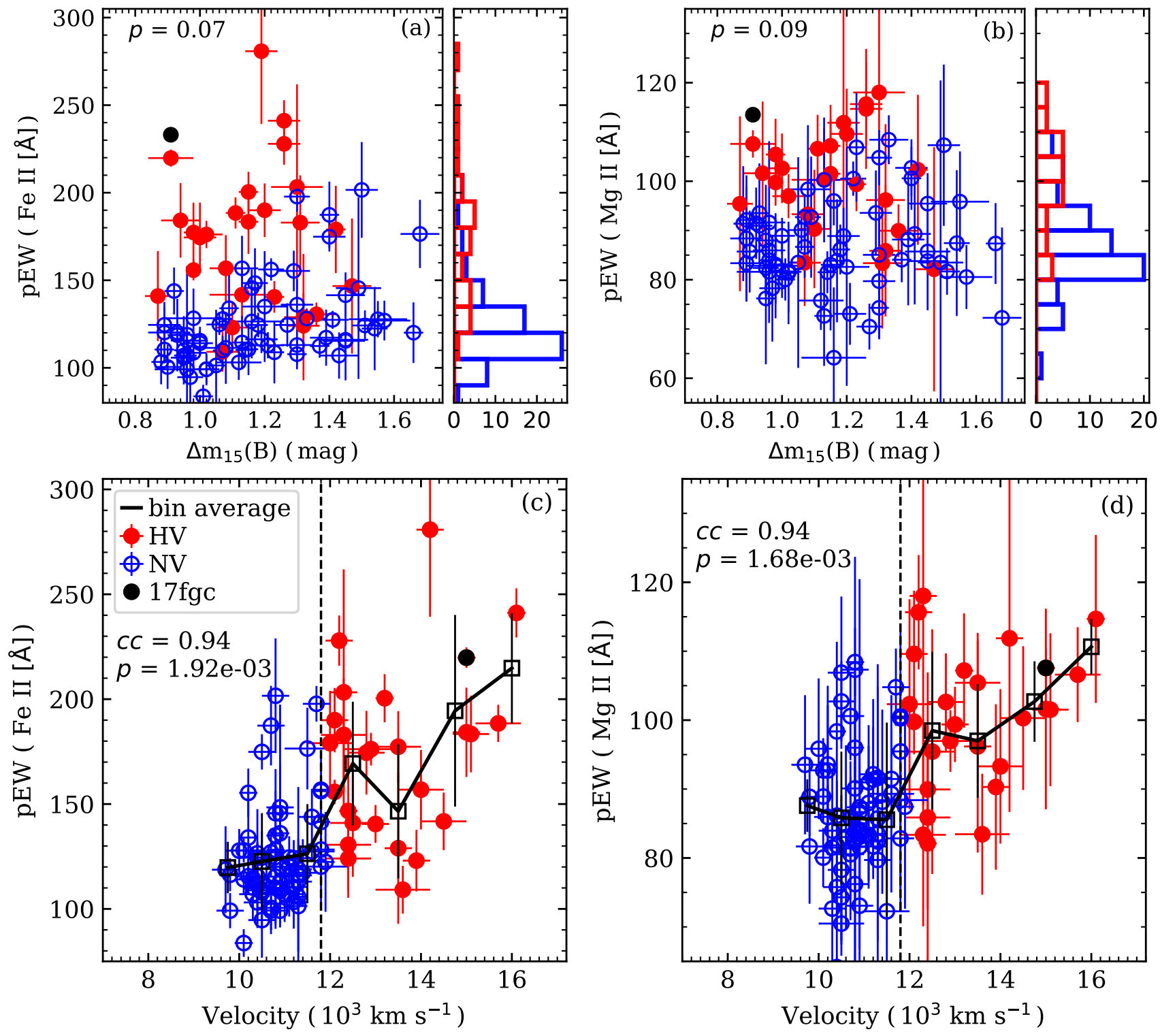

Figure 19. Panels (a) and (b): The pseudo-equivalent widths (pEWs) of blended absorptions of Fe III $\lambda 5129, \mathrm{Fe}$ II $\lambda \lambda \lambda 4924,5018,5169$, and Si II $\lambda 5051$ measured in the near-maximum-light spectra are plotted versus the luminosity indicator $\Delta m_{15}(B)$ for $92 \mathrm{SNe}$ Ia from Wang et al. (2019b). The $p$ represents the p-value (with significance level $\sim 2 \sigma$ ) from the $T$-test statistics. Panels (c) and (d): The pEWs of the blended absorptions of Fe II $\lambda 4404$ and $\mathrm{Mg}$ II $\lambda 4481$ are plotted as a function of Si II velocity obtained around $B$-band maximum. The HV SNe Ia are represented with red dots while the NV subclass are blue open circles. For comparison, SN 2017fgc is shown with a black dot. The Si II velocities of SNe Ia are taken from Wang et al. (2019b). The black open square with black line represents the binned average, and $c c$ and $p$ are the correlation coefficient and p-value from pearson statistics (with significance level $\sim 3 \sigma$ ), respectively. The vertical dashed lines represent the boundary between the HV and NV SNe Ia as defined by Wang et al. (2009b).

fusion model (Arnett 1982), we find that SN 2017fgc has a peak luminosity of $L_{\text {peak }}=(1.32 \pm 0.13) \times 10^{43} \mathrm{erg} \mathrm{s}^{-1}$ and a synthesized nickel mass of $M_{\mathrm{Ni}}=0.51 \pm 0.03 M_{\odot}$.

The light curve and color curve evolution of SN $2017 \mathrm{fgc}$ are similar to those of other HV SNe Ia such as SN 2002bo and SN 2006X. The relatively bright tails in the $U$ and $B$ light curves observed in SN 2017fgc may be indicators of CSM around the progenitor system. Its spectral evolution is similar to those of SN 2002bo, SN 2006X, and SN 2013gs, with an unusally high Si II velocity near maximum light $\left(\sim 15,000 \pm 150 \mathrm{~km} \mathrm{~s}^{-1}\right)$ as well as stronger absorptions of $\mathrm{Fe}$ II/Mg II blends near $4400 \AA$ and $\mathrm{Fe}$ III/Fe II/Si II blends near $4900 \AA$ A. Moreover, SN 2017 fgc and other HV SNe Ia are found to have more pronounced secondary maximum peaks in the $I i$ bands. All of the above features indicate that SN $2017 \mathrm{fgc}$ and other HV SNe Ia likely have experienced more complete burning, or their progenitors have higher metallicity environments. 


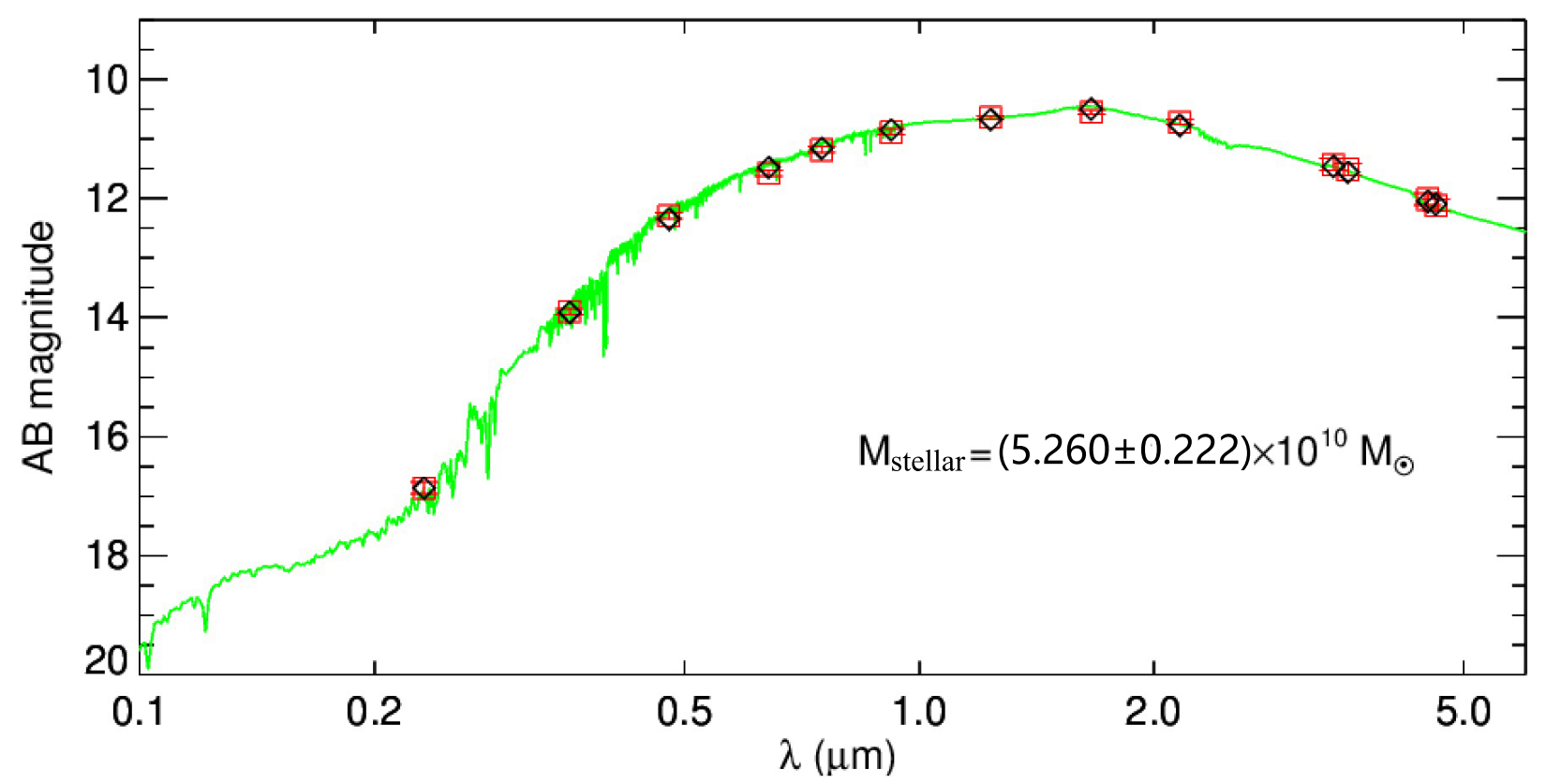

Figure 20. The stellar population synthesis fit for the SED of NGC474. The red open squares represent the observed AB magnitudes, while the synthesized magnitudes from the best-fit spectrum template (green curve) are overplotted as black diamonds.

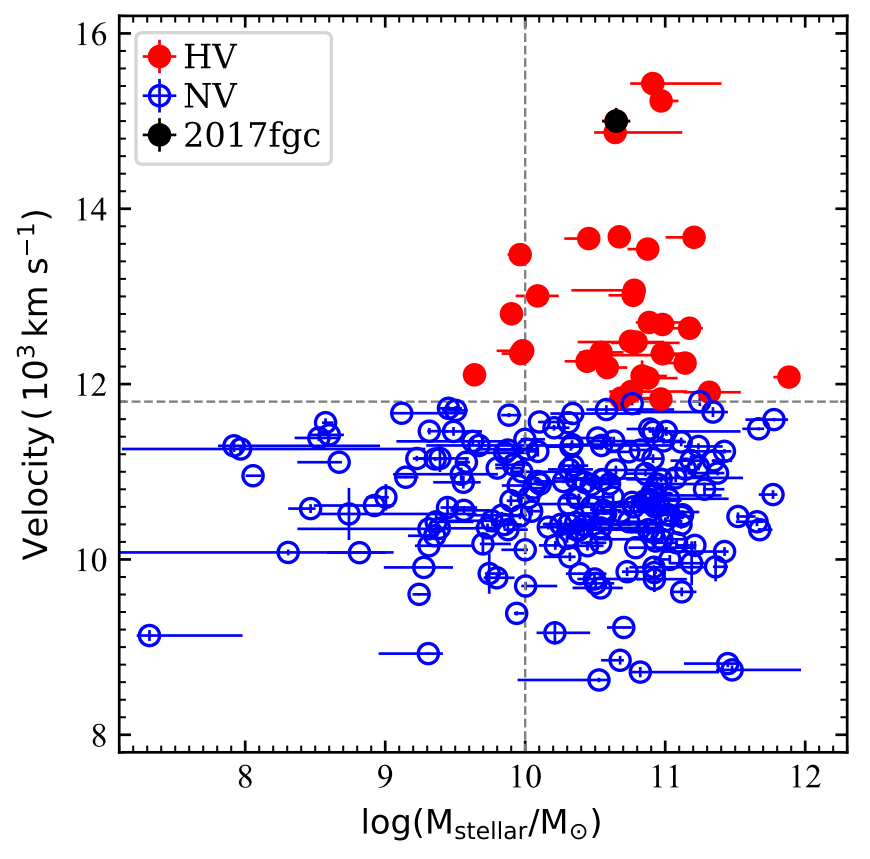

Figure 21. Scatter plot of the Si II $\lambda 6355$ velocities and the corresponding host-galaxy stellar mass $\left(M_{\text {stellar }}\right)$. The HV SNe Ia are shown as red dots, and the NV SNe Ia are shown as blue circles. The horizontal and vertical dashed lines represent the criteria used to split the sample in velocity (Wang et al. 2009a) and $M_{\text {stellar }}$ (Pan 2020) space, respectively. The data are taken from Pan (2020) and SN 2017 fgc (this paper) is overplotted as a black dot.

Inspection of its birthplace environment indicates that $\mathrm{SN}$ $2017 \mathrm{fgc}$ was born in a gas bridge with young and metal-rich stellar populations. However, the fact that it was located far away from the center of its host galaxy indicates that its progenitor cannot be metal rich. A possible scenario is that the progenitor of SN 2017fgc could be ejected from the inner part of the companion galaxy during the merger $\sim 2 \mathrm{Gyr}$ ago, or formed as a result of some cold gas remained in the companion disk (Alabi et al. 2020; Fensch et al. 2020). Detailed study of the host environment of SN 2017fgc is needed. Also, more observations and further modeling are essential to reveal the origin of the strong absorption of Fe II and IMEs seen in SN 2017fgc and the nature of the fast-expanding subclass of SNe Ia.

\section{ACKNOWLEDGMENTS}

We thank the anonymous referee for his/her suggestive comments that help improve the manuscript a lot. This work is supported by the National Natural Science Foundation of China (NSFC, grants 11873081, U2031209, 12033002, 11633002, 11803076, and 11761141001), the National Program on Key Research and Development Project (grant 2016YFA0400803), and the High Level Talent-Heaven Lake Program of Xinjiang Uygur Autonomous Region of China. Moreover, the Scholar Program of the Beijing Academy of Science and Technology (DZ:BS202002) provides partial support for the work. Staffs of the Lijiang $2.4 \mathrm{~m}$ telescope (LJT), the Xinglong $2.16 \mathrm{~m}$ telescope (XLT), and Lick Observatory assisted with the observations. The Chinese Academy of Sciences and the People's Government of Yunnan Province provide support for the LJT, which is corporately run and maintained by Yunnan Observatories and 
the Center for Astronomical Mega-Science (CAS). JuJia Zhang is supported by the National Natural Science Foundation of China (NSFC; grants 11773067 and 11403096), the Youth Innovation Promotion Association of the CAS (grant 2018081), and the Ten Thousand Talents Program of Yunnan for Top-notch Young Talents. Support for A.V.F.'s group at U.C. Berkeley was provided by the TABASGO Foundation, the Christopher R. Redlich Fund, and the Miller Institute for Basic Research in Science. Research by D.J.S. is supported by the U.S. National Science Foundation (NSF) grants AST$1821967,1821987,1813708,1813466,1908972$, and by the Heising-Simons Foundation under grant \#2020-1864. J.B., D.H., D.A.H., and C.P. were supported by NSF grant AST1911225. This work makes use of data from the Las Cumbres Observatory network.

Some of the observations with the Lick Observatory $1 \mathrm{~m}$ Nickel telescope were conducted by U.C. Berkeley undergraduate students Sanyum Channa, Edward Falcon, Nachiket Girish, Romain Hardy, Julia Hestenes, Andrew Hoffman, Evelyn Liu, Shaunak Modak, Costas Soler, Kevin Tang, Sameen Yunus, and Keto Zhang; we thank them for their excellent work. Lick/KAIT and its ongoing operation were made possible by donations from Sun Microsystems, Inc., the Hewlett-Packard Company, AutoScope Corporation, Lick Observatory, the NSF, the University of California, the Sylvia \& Jim Katzman Foundation, and the TABASGO Foundation. A major upgrade of the Kast spectrograph on the Shane $3 \mathrm{~m}$ telescope at Lick Observatory was made possible through generous gifts from the Heising-Simons Foundation as well as William and Marina Kast. Research at Lick Observatory is partially supported by a generous gift from Google. This research has made use of the services of the ESO Science Archive Facility.

Software: SNooPy2 (Burns et al. 2011, 2014), SALT 2.4 (Guy et al. 2010; Betoule et al. 2014), LOSSPhotPypeline (https://github.com/benstahl92/LOSSPhotPypeline), SN-Spectral Evolution (https://github.com/mwvgroup/SNSpectral-Evolution), Minim Code (Chatzopoulos et al. 2013), IRAF (Tody 1993, 1986), DAOPHOT (Stetson 1987), Photutils (Bradley et al. 2020), lcogtsnpipe (Valenti et al. 2016), respext(https://github.com/benstahl92/respext), Astropy (Astropy Collaboration et al. 2013), Matplotlib (Hunter 2007), Scipy (https://www.scipy.org/), Numpy (https://numpy.org/) 


\section{REFERENCES}

Alabi, A. B., Ferré-Mateu, A., Forbes, D. A., Romanowsky, A. J., \& Brodie, J. P. 2020, MNRAS, 497, 626,

doi: 10.1093/mnras/staa1992

Alam, S., Albareti, F. D., Allende Prieto, C., et al. 2015, ApJS, 219, 12, doi: 10.1088/0067-0049/219/1/12

Aldering, G., Antilogus, P., Bailey, S., et al. 2006, ApJ, 650, 510, doi: 10.1086/507020

Arnett, W. D. 1982, ApJ, 253, 785, doi: 10.1086/159681

Astropy Collaboration, Robitaille, T. P., Tollerud, E. J., et al. 2013, A\&A, 558, A33, doi: 10.1051/0004-6361/201322068

Barbon, R., Iijima, T., \& Rosino, L. 1989, A\&A, 220, 83

Benetti, S., Meikle, P., Stehle, M., et al. 2004, MNRAS, 348, 261, doi: 10.1111/j.1365-2966.2004.07357.x

Benetti, S., Cappellaro, E., Mazzali, P. A., et al. 2005, ApJ, 623, 1011, doi: 10.1086/428608

Betoule, M., Kessler, R., Guy, J., et al. 2014, A\&A, 568, A22, doi: 10.1051/0004-6361/201423413

Blondin, S., Dessart, L., \& Hillier, D. J. 2015, MNRAS, 448, 2766, doi: 10.1093/mnras/stv188

Blondin, S., Prieto, J. L., Patat, F., et al. 2009, ApJ, 693, 207, doi: 10.1088/0004-637X/693/1/207

Blondin, S., Matheson, T., Kirshner, R. P., et al. 2012, AJ, 143, 126, doi: 10.1088/0004-6256/143/5/126

Bochenek, C. D., Dwarkadas, V. V., Silverman, J. M., et al. 2018, MNRAS, 473, 336, doi: 10.1093/mnras/stx2029

Bradley, L., Sipőcz, B., Robitaille, T., et al. 2020, astropy/photutils: 1.0.0, 1.0.0, Zenodo, doi: 10.5281/zenodo.4044744

Branch, D., Fisher, A., \& Nugent, P. 1993, AJ, 106, 2383, doi: $10.1086 / 116810$

Branch, D., Dang, L. C., Hall, N., et al. 2006, PASP, 118, 560, doi: 10.1086/502778

Bravo, E., \& García-Senz, D. 2008, A\&A, 478, 843, doi: 10.1051/0004-6361:20078424

Brown, T. M., Burleson, B., Crellin, M., et al. 2010, in American Astronomical Society Meeting Abstracts, Vol. 215, American Astronomical Society Meeting Abstracts \#215, 441.06

Brown, T. M., Baliber, N., Bianco, F. B., et al. 2013, PASP, 125, 1031, doi: 10.1086/673168

Bruzual, G., \& Charlot, S. 2003, MNRAS, 344, 1000, doi: 10.1046/j.1365-8711.2003.06897.x

Burgaz, U., Maeda, K., Kalomeni, B., et al. 2021, MNRAS, 502, 4112, doi: 10.1093/mnras/stab254

Burns, C. R., Stritzinger, M., Phillips, M. M., et al. 2011, AJ, 141, 19, doi: 10.1088/0004-6256/141/1/19

—. 2014, ApJ, 789, 32, doi: 10.1088/0004-637X/789/1/32

Burns, C. R., Parent, E., Phillips, M. M., et al. 2018, ApJ, 869, 56, doi: 10.3847/1538-4357/aae51c

Cappellari, M., Emsellem, E., Krajnović, D., et al. 2011, MNRAS, 413, 813, doi: 10.1111/j.1365-2966.2010.18174.x
Cardelli, J. A., Clayton, G. C., \& Mathis, J. S. 1989, ApJ, 345, 245 , doi: 10.1086/167900

Cartier, R., Hamuy, M., Pignata, G., et al. 2014, ApJ, 789, 89, doi: 10.1088/0004-637X/789/1/89

Chabrier, G. 2003, PASP, 115, 763, doi: 10.1086/376392

Chakradhari, N. K., Sahu, D. K., \& Anupama, G. C. 2019, MNRAS, 487, 1886, doi: 10.1093/mnras/stz1358

Chatzopoulos, E., Wheeler, J. C., \& Vinko, J. 2012, ApJ, 746, 121, doi: 10.1088/0004-637X/746/2/121

Chatzopoulos, E., Wheeler, J. C., Vinko, J., Horvath, Z. L., \& Nagy, A. 2013, ApJ, 773, 76, doi: 10.1088/0004-637X/773/1/76

Chen, D., Wang, J.-C., Xu, J., et al. 2001, Publications of the Yunnan Observatory, 4, 42

Cikota, A., Patat, F., Wang, L., et al. 2019, MNRAS, 490, 578, doi: 10.1093/mnras/stz2322

Dessart, L., Hillier, D. J., Blondin, S., \& Khokhlov, A. 2014, MNRAS, 441, 3249, doi: 10.1093/mnras/stu789

Dilday, B., Howell, D. A., Cenko, S. B., et al. 2012, Science, 337, 942, doi: 10.1126/science.1219164

Domínguez, I., Höflich, P., \& Straniero, O. 2001, ApJ, 557, 279, doi: $10.1086 / 321661$

Duc, P.-A., Cuillandre, J.-C., Karabal, E., et al. 2015, MNRAS, 446, 120, doi: 10.1093/mnras/stu2019

Fan, Z., Wang, H., Jiang, X., et al. 2016, PASP, 128, 115005 , doi: 10.1088/1538-3873/128/969/115005

Fensch, J., Duc, P.-A., Lim, S., et al. 2020, A\&A, 644, A164, doi: 10.1051/0004-6361/202038550

Filippenko, A. V. 1997, ARA\&A, 35, 309, doi: 10.1146/annurev.astro.35.1.309

Filippenko, A. V. 2003, in From Twilight to Highlight: The Physics of Supernovae, ed. W. Hillebrandt \& B. Leibundgut, 171, doi: 10.1007/10828549_23

Filippenko, A. V., Chornock, R. T., \& Li, W. D. 1999, IAUC, 7272, 3

Filippenko, A. V., Ebneter, K., Kirshner, R. P., \& Winkler, P. F. 1986, IAUC, 4190, 2

Filippenko, A. V., Li, W. D., Treffers, R. R., \& Modjaz, M. 2001, in Astronomical Society of the Pacific Conference Series, Vol. 246, IAU Colloq. 183: Small Telescope Astronomy on Global Scales, ed. B. Paczynski, W.-P. Chen, \& C. Lemme, 121

Filippenko, A. V., Richmond, M. W., Matheson, T., et al. 1992a, ApJL, 384, L15, doi: 10.1086/186252

Filippenko, A. V., Richmond, M. W., Branch, D., et al. 1992b, AJ, 104, 1543, doi: 10.1086/116339

Folatelli, G., Morrell, N., Phillips, M. M., et al. 2013, ApJ, 773, 53, doi: 10.1088/0004-637X/773/1/53

Foley, R. J., Challis, P. J., Filippenko, A. V., et al. 2012, ApJ, 744, 38, doi: 10.1088/0004-637X/744/1/38 
Foley, R. J., Challis, P. J., Chornock, R., et al. 2013, ApJ, 767, 57, doi: 10.1088/0004-637X/767/1/57

Ganeshalingam, M., Li, W., \& Filippenko, A. V. 2011, MNRAS, 416, 2607, doi: 10.1111/j.1365-2966.2011.19213.x

Ganeshalingam, M., Li, W., Filippenko, A. V., et al. 2010, ApJS, 190, 418, doi: 10.1088/0067-0049/190/2/418

Gerardy, C. L., Höflich, P., Fesen, R. A., et al. 2004, ApJ, 607, 391, doi: $10.1086 / 383488$

González Hernández, J. I., Ruiz-Lapuente, P., Tabernero, H. M., et al. 2012, Nature, 489, 533, doi: 10.1038/nature11447

Graur, O., Maguire, K., Ryan, R., et al. 2020, Nature Astronomy, 4, 188, doi: 10.1038/s41550-019-0901-1

Gutiérrez, C. P., González-Gaitán, S., Folatelli, G., et al. 2016, A\&A, 590, A5, doi: 10.1051/0004-6361/201527228

Guy, J., Astier, P., Nobili, S., Regnault, N., \& Pain, R. 2005, A\&A, 443, 781, doi: 10.1051/0004-6361:20053025

Guy, J., Sullivan, M., Conley, A., et al. 2010, A\&A, 523, A7, doi: 10.1051/0004-6361/201014468

Hamuy, M., Phillips, M. M., Suntzeff, N. B., et al. 2003, Nature, 424, 651, doi: 10.1038/nature01854

Han, X., Zheng, W., Stahl, B. E., et al. 2020, ApJ, 892, 142, doi: 10.3847/1538-4357/ab7a27

Hernández-Toledo, H. M., Méndez-Hernández, H., Aceves, H., \& Olguín, L. 2011, AJ, 141, 74, doi: 10.1088/0004-6256/141/3/74

Hicken, M., Challis, P., Kirshner, R. P., et al. 2012, ApJS, 200, 12, doi: 10.1088/0067-0049/200/2/12

Hillebrandt, W., \& Niemeyer, J. C. 2000, ARA\&A, 38, 191, doi: 10.1146/annurev.astro.38.1.191

Höflich, P. 1995, ApJ, 443, 89, doi: 10.1086/175505

Höflich, P., Gerardy, C. L., Fesen, R. A., \& Sakai, S. 2002, ApJ, 568, 791, doi: 10.1086/339063

Höflich, P., Gerardy, C. L., Marion, H., \& Quimby, R. 2006, NewAR, 50, 470, doi: 10.1016/j.newar.2006.06.074

Howell, D. A. 2011, Nature Communications, 2, 350, doi: 10.1038/ncomms1344

Howell, D. A., Sullivan, M., Nugent, P. E., et al. 2006, Nature, 443, 308, doi: 10.1038/nature05103

Huang, F., Li, J.-Z., Wang, X.-F., et al. 2012, Research in Astronomy and Astrophysics, 12, 1585, doi: 10.1088/1674-4527/12/11/012

Hunter, J. D. 2007, Computing in Science Engineering, 9, 90, doi: 10.1109/MCSE.2007.55

Iben, I., J., \& Tutukov, A. V. 1984, ApJS, 54, 335, doi: $10.1086 / 190932$

Jack, D., Hauschildt, P. H., \& Baron, E. 2012, A\&A, 538, A132, doi: 10.1051/0004-6361/201117271

Jha, S. W., Maguire, K., \& Sullivan, M. 2019, Nature Astronomy, 3, 706, doi: 10.1038/s41550-019-0858-0

Jiang, X., Xu, D., \& Hu, J. 1999, Acta Astrophysica Sinica, 19, 220
Jordi, K., Grebel, E. K., \& Ammon, K. 2006, A\&A, 460, 339, doi: 10.1051/0004-6361:20066082

Kasen, D. 2006, ApJ, 649, 939, doi: 10.1086/506588

—. 2010, ApJ, 708, 1025, doi: 10.1088/0004-637X/708/2/1025

Kasen, D., \& Woosley, S. E. 2007, ApJ, 656, 661, doi: $10.1086 / 510375$

Kawabata, M., Maeda, K., Yamanaka, M., et al. 2020, ApJ, 893, 143, doi: 10.3847/1538-4357/ab8236

Khokhlov, A. 2005, in APS Meeting Abstracts, Vol. 47, APS Division of Plasma Physics Meeting Abstracts, CZ2.006

Khokhlov, A. M. 1989, MNRAS, 239, 785, doi: 10.1093/mnras/239.3.785

Krisciunas, K., Suntzeff, N. B., Phillips, M. M., et al. 2004, AJ, 128, 3034, doi: 10.1086/425629

Landolt, A. U. 1992, AJ, 104, 340, doi: 10.1086/116242

Laurent, F., Adjali, L., Arns, J., et al. 2010, in Society of Photo-Optical Instrumentation Engineers (SPIE) Conference Series, Vol. 7739, Modern Technologies in Space- and Ground-based Telescopes and Instrumentation, ed. E. Atad-Ettedgui \& D. Lemke, 77394M, doi: 10.1117/12.857004

Leibundgut, B., Kirshner, R. P., Phillips, M. M., et al. 1993, AJ, 105, 301, doi: 10.1086/116427

Levanon, N., \& Soker, N. 2017, MNRAS, 470, 2510, doi: 10.1093/mnras/stx1387

Li, W., Filippenko, A. V., Chornock, R., et al. 2003, PASP, 115, 453, doi: 10.1086/374200

Li, W., Leaman, J., Chornock, R., et al. 2011, MNRAS, 412, 1441, doi: 10.1111/j.1365-2966.2011.18160.x

Li, W., Wang, X., Vinkó, J., et al. 2019, ApJ, 870, 12, doi: 10.3847/1538-4357/aaec74

Li, W., Wang, X., Bulla, M., et al. 2021, ApJ, 906, 99, doi: 10.3847/1538-4357/abc9b5

Lim, S., Peng, E. W., Duc, P.-A., et al. 2017, ApJ, 835, 123, doi: 10.3847/1538-4357/835/2/123

Lin, L., Zou, H., Kong, X., et al. 2013, ApJ, 769, 127, doi: 10.1088/0004-637X/769/2/127

Livio, M., \& Mazzali, P. 2018, PhR, 736, 1, doi: 10.1016/j.physrep.2018.02.002

Maeda, K., Benetti, S., Stritzinger, M., et al. 2010, Nature, 466, 82, doi: 10.1038/nature09122

Maguire, K., Sim, S. A., Shingles, L., et al. 2018, MNRAS, 477, 3567, doi: 10.1093/mnras/sty820

Maoz, D., Mannucci, F., \& Nelemans, G. 2014, ARA\&A, 52, 107, doi: 10.1146/annurev-astro-082812-141031

Marion, G. H., Vinko, J., Wheeler, J. C., et al. 2013, ApJ, 777, 40, doi: 10.1088/0004-637X/777/1/40

Munari, U., Henden, A., Belligoli, R., et al. 2013, NewA, 20, 30, doi: 10.1016/j.newast.2012.09.003

Noebauer, U. M., Kromer, M., Taubenberger, S., et al. 2017, MNRAS, 472, 2787, doi: 10.1093/mnras/stx2093 
Nomoto, K., Iwamoto, K., \& Kishimoto, N. 1997, Science, 276, 1378, doi: 10.1126/science.276.5317.1378

Nomoto, K., Thielemann, F. K., \& Yokoi, K. 1984, ApJ, 286, 644, doi: $10.1086 / 162639$

Nordin, J., Östman, L., Goobar, A., et al. 2011, A\&A, 526, A119, doi: 10.1051/0004-6361/201015705

Nugent, P., Phillips, M., Baron, E., Branch, D., \& Hauschildt, P. 1995, ApJL, 455, L147, doi: 10.1086/309846

Olling, R. P., Mushotzky, R., Shaya, E. J., et al. 2015, Nature, 521, 332, doi: 10.1038/nature14455

Pakmor, R., Kromer, M., Taubenberger, S., et al. 2012, ApJL, 747, L10, doi: 10.1088/2041-8205/747/1/L10

Pan, Y.-C. 2020, ApJL, 895, L5, doi: 10.3847/2041-8213/ab8e47

Pan, Y. C., Sullivan, M., Maguire, K., et al. 2015, MNRAS, 446, 354, doi: 10.1093/mnras/stu2121

Pastorello, A., Mazzali, P. A., Pignata, G., et al. 2007, MNRAS, 377, 1531, doi: 10.1111/j.1365-2966.2007.11700.x

Perlmutter, S., Aldering, G., Goldhaber, G., et al. 1999, ApJ, 517, 565, doi: 10.1086/307221

Phillips, M. M. 1993, ApJL, 413, L105, doi: 10.1086/186970

Phillips, M. M., Lira, P., Suntzeff, N. B., et al. 1999, AJ, 118, 1766, doi: $10.1086 / 301032$

Pignata, G., Benetti, S., Mazzali, P. A., et al. 2008, MNRAS, 388, 971, doi: 10.1111/j.1365-2966.2008.13434.x

Pinto, P. A., \& Eastman, R. G. 2000, ApJ, 530, 757, doi: $10.1086 / 308380$

Piro, A. L., \& Morozova, V. S. 2016, ApJ, 826, 96, doi: 10.3847/0004-637X/826/1/96

Piro, A. L., \& Nakar, E. 2013, ApJ, 769, 67, doi: 10.1088/0004-637X/769/1/67

Podsiadlowski, P., Mazzali, P., Lesaffre, P., Han, Z., \& Förster, F. 2008, NewAR, 52, 381, doi: 10.1016/j.newar.2008.06.020

Polin, A., Nugent, P., \& Kasen, D. 2019, ApJ, 873, 84, doi: 10.3847/1538-4357/aafb6a

Rasio, F. A., \& Shapiro, S. L. 1994, ApJ, 432, 242, doi: $10.1086 / 174566$

Raskin, C., \& Kasen, D. 2013, ApJ, 772, 1, doi: 10.1088/0004-637X/772/1/1

Raskin, C., Kasen, D., Moll, R., Schwab, J., \& Woosley, S. 2014, ApJ, 788, 75, doi: 10.1088/0004-637X/788/1/75

Riess, A. G., Press, W. H., \& Kirshner, R. P. 1996, ApJ, 473, 88, doi: $10.1086 / 178129$

Riess, A. G., Filippenko, A. V., Challis, P., et al. 1998, AJ, 116, 1009, doi: 10.1086/300499

Riess, A. G., Filippenko, A. V., Li, W., et al. 1999, AJ, 118, 2675, doi: 10.1086/301143

Riess, A. G., Casertano, S., Yuan, W., et al. 2018, ApJ, 855, 136, doi: 10.3847/1538-4357/aaadb7

Röpke, F. K., \& Niemeyer, J. C. 2007, A\&A, 464, 683, doi: 10.1051/0004-6361:20066585
Röpke, F. K., Kromer, M., Seitenzahl, I. R., et al. 2012, ApJL, 750, L19, doi: 10.1088/2041-8205/750/1/L19

Ruiz-Lapuente, P., Cappellaro, E., Turatto, M., et al. 1992, ApJL, 387, L33, doi: 10.1086/186299

Sahu, D. K., Anupama, G. C., \& Anto, P. 2013, MNRAS, 430, 869, doi: 10.1093/mnras/sts609

Sand, D. J., Brown, T., Haynes, R., \& Dubberley, M. 2011, in American Astronomical Society Meeting Abstracts, Vol. 218, American Astronomical Society Meeting Abstracts \#218, 132.03

Sato, Y., Nakasato, N., Tanikawa, A., et al. 2015, ApJ, 807, 105, doi: 10.1088/0004-637X/807/1/105

Schaefer, B. E., \& Pagnotta, A. 2012, Nature, 481, 164, doi: 10.1038/nature10692

Schlafly, E. F., \& Finkbeiner, D. P. 2011, ApJ, 737, 103, doi: 10.1088/0004-637X/737/2/103

Schlegel, D. J., Finkbeiner, D. P., \& Davis, M. 1998, ApJ, 500, 525, doi: 10.1086/305772

Scolnic, D. M., Jones, D. O., Rest, A., et al. 2018, ApJ, 859, 101, doi: 10.3847/1538-4357/aab9bb

Seitenzahl, I. R., Ciaraldi-Schoolmann, F., Röpke, F. K., et al. 2013, MNRAS, 429, 1156, doi: 10.1093/mnras/sts402

Shen, K. J., Guillochon, J., \& Foley, R. J. 2013, ApJL, 770, L35, doi: 10.1088/2041-8205/770/2/L35

Silverman, J. M., \& Filippenko, A. V. 2012, MNRAS, 425, 1917, doi: 10.1111/j.1365-2966.2012.21276.x

Silverman, J. M., Ganeshalingam, M., Li, W., \& Filippenko, A. V. 2012a, MNRAS, 425, 1889, doi: 10.1111/j.1365-2966.2012.21526.x

Silverman, J. M., Kong, J. J., \& Filippenko, A. V. 2012b, MNRAS, 425, 1819, doi: 10.1111/j.1365-2966.2012.21269.x

Silverman, J. M., Nugent, P. E., Gal-Yam, A., et al. 2013, ApJ, 772, 125, doi: 10.1088/0004-637X/772/2/125

Soker, N. 2019, NewAR, 87, 101535, doi: 10.1016/j.newar.2020.101535

Stahl, B. E., Martínez-Palomera, J., Zheng, W., et al. 2020a, MNRAS, 496, 3553, doi: 10.1093/mnras/staa1706

Stahl, B. E., Zheng, W., de Jaeger, T., et al. 2019, MNRAS, 490, 3882, doi: 10.1093/mnras/stz2742

—. 2020b, MNRAS, 492, 4325, doi: 10.1093/mnras/staa102

Stanishev, V., Goobar, A., Benetti, S., et al. 2007, A\&A, 469, 645, doi: 10.1051/0004-6361:20066020

Sternberg, A., Gal-Yam, A., Simon, J. D., et al. 2011a, Science, 333, 856, doi: 10.1126/science. 1203836

—. 2011b, Science, 333, 856, doi: 10.1126/science. 1203836

Stetson, P. B. 1987, PASP, 99, 191, doi: 10.1086/131977

—. 2000, PASP, 112, 925, doi: 10.1086/316595

Stritzinger, M. D., Phillips, M. M., Boldt, L. N., et al. 2011, AJ, 142, 156, doi: 10.1088/0004-6256/142/5/156

Taddia, F., Stritzinger, M. D., Phillips, M. M., et al. 2012, A\&A, 545, L7, doi: 10.1051/0004-6361/201220105 
Tartaglia, L., Sand, D. J., Valenti, S., et al. 2018a, ApJ, 853, 62, doi: 10.3847/1538-4357/aaa014

—. 2018b, ApJ, 853, 62, doi: 10.3847/1538-4357/aaa014

Thompson, T. A. 2011, ApJ, 741, 82, doi: 10.1088/0004-637X/741/2/82

Timmes, F. X., Brown, E. F., \& Truran, J. W. 2003, ApJL, 590, L83, doi: 10.1086/376721

Tody, D. 1986, in Society of Photo-Optical Instrumentation Engineers (SPIE) Conference Series, Vol. 627, Instrumentation in astronomy VI, ed. D. L. Crawford, 733, doi: $10.1117 / 12.968154$

Tody, D. 1993, in Astronomical Society of the Pacific Conference Series, Vol. 52, Astronomical Data Analysis Software and Systems II, ed. R. J. Hanisch, R. J. V. Brissenden, \& J. Barnes, 173

Tucker, M. A., Shappee, B. J., \& Wisniewski, J. P. 2019, ApJL, 872, L22, doi: 10.3847/2041-8213/ab0286

Tully, R. B., Courtois, H. M., Dolphin, A. E., et al. 2013, AJ, 146, 86, doi: 10.1088/0004-6256/146/4/86

Valenti, S., Sand, D., Tartaglia, L., et al. 2017a, Transient Name Server Classification Report, 2017-757, 1

Valenti, S., Sand, D. J., \& Tartaglia, L. 2017b, Transient Name Server Discovery Report, 2017-753, 1

Valenti, S., Howell, D. A., Stritzinger, M. D., et al. 2016, MNRAS, 459, 3939, doi: 10.1093/mnras/stw870

Vernet, J., Dekker, H., D’Odorico, S., et al. 2011, A\&A, 536, A105, doi: 10.1051/0004-6361/201117752

Vogt, S. S. 1987, in Liege International Astrophysical Colloquia, Vol. 27, Liege International Astrophysical Colloquia, ed. J. P. Swings, J. Collin, \& E. J. Wampler, 345-351

Walker, E. S., Hook, I. M., Sullivan, M., et al. 2011, MNRAS, 410, 1262, doi: 10.1111/j.1365-2966.2010.17519.x

Wang, C.-J., Bai, J.-M., Fan, Y.-F., et al. 2019a, Research in Astronomy and Astrophysics, 19, 149, doi: 10.1088/1674-4527/19/10/149

Wang, L., Baade, D., Höflich, P., et al. 2004, ApJL, 604, L53, doi: 10.1086/383411

Wang, L., Baade, D., \& Patat, F. 2007, Science, 315, 212, doi: 10.1126/science.1121656

Wang, X., Chen, J., Wang, L., et al. 2019b, ApJ, 882, 120, doi: 10.3847/1538-4357/ab26b5

Wang, X., Li, W., Filippenko, A. V., et al. 2008a, ApJ, 677, 1060, doi: $10.1086 / 529070$

Wang, X., Wang, L., Filippenko, A. V., Zhang, T., \& Zhao, X. 2013, Science, 340, 170, doi: 10.1126/science.1231502

Wang, X., Wang, L., Pain, R., Zhou, X., \& Li, Z. 2006, ApJ, 645, 488, doi: $10.1086 / 504312$
Wang, X., Wang, L., Zhou, X., Lou, Y.-Q., \& Li, Z. 2005, ApJL, 620, L87, doi: 10.1086/428774

Wang, X., Li, W., Filippenko, A. V., et al. 2008b, ApJ, 675, 626, doi: $10.1086 / 526413$

Wang, X., Filippenko, A. V., Ganeshalingam, M., et al. 2009a, ApJL, 699, L139, doi: 10.1088/0004-637X/699/2/L139

Wang, X., Li, W., Filippenko, A. V., et al. 2009b, ApJ, 697, 380, doi: 10.1088/0004-637X/697/1/380

Webbink, R. F. 1984, ApJ, 277, 355, doi: 10.1086/161701

Wei, P., Zou, H., Lin, L., et al. 2021, Research in Astronomy and Astrophysics, 21, 006, doi: 10.1088/1674-4527/21/1/6

Whelan, J., \& Iben, Icko, J. 1973, ApJ, 186, 1007, doi: $10.1086 / 152565$

Woosley, S. E., Kerstein, A. R., Sankaran, V., Aspden, A. J., \& Röpke, F. K. 2009, ApJ, 704, 255, doi: 10.1088/0004-637X/704/1/255

Wu, J. F., \& Boada, S. 2019, MNRAS, 484, 4683, doi: $10.1093 / \mathrm{mnras} / \mathrm{stz} 333$

Yamanaka, M., Naito, H., Kinugasa, K., et al. 2009, PASJ, 61, 713, doi: 10.1093/pasj/61.4.713

Yang, Y., Wang, L., Baade, D., et al. 2018, ApJ, 854, 55, doi: $10.3847 / 1538-4357 /$ aaa76a

Yoon, S. C., Podsiadlowski, P., \& Rosswog, S. 2007, MNRAS, 380, 933, doi: 10.1111/j.1365-2966.2007.12161.X

Zeng, X., Wang, X., Esamdin, A., et al. 2021, ApJ, 909, 176, doi: 10.3847/1538-4357/abdeb9

Zhang, J.-C., Fan, Z., Yan, J.-Z., et al. 2016a, PASP, 128, 105004, doi: 10.1088/1538-3873/128/968/105004

Zhang, K., Wang, X., Zhang, J., et al. 2016b, ApJ, 820, 67, doi: 10.3847/0004-637X/820/1/67

Zhang, T., Wang, X., Li, W., et al. 2010, PASP, 122, 1, doi: 10.1086/649851

Zhang, T., Wang, X., Zhao, X., et al. 2019, ApJ, 872, 14, doi: 10.3847/1538-4357/aafacd

Zhao, X., Wang, X., Maeda, K., et al. 2015, ApJS, 220, 20, doi: 10.1088/0067-0049/220/1/20

Zhao, X., Maeda, K., Wang, X., et al. 2016, ApJ, 826, 211, doi: 10.3847/0004-637X/826/2/211

Zheng, W., Kelly, P. L., \& Filippenko, A. V. 2017a, ApJ, 848, 66, doi: 10.3847/1538-4357/aa8b19

—. 2018, ApJ, 858, 104, doi: 10.3847/1538-4357/aabaeb

Zheng, W., Filippenko, A. V., Mauerhan, J., et al. 2017b, ApJ, 841, 64, doi: 10.3847/1538-4357/aa6dfa 
Table 1. Photometric Standards in the SN 2017 fgc Field $1^{a}$

\begin{tabular}{|c|c|c|c|c|c|c|c|c|c|c|}
\hline Star & $\alpha(\mathrm{J} 2000)$ & $\delta(\mathrm{J} 2000)$ & $U$ (mag) & $B$ (mag) & $V$ (mag) & $R$ (mag) & $I$ (mag) & $g$ (mag) & $r(\mathrm{mag})$ & $i$ (mag) \\
\hline 1 & & & & & & & & 16.31 & & \\
\hline 2 & & & & & & & & & & \\
\hline 3 & :19:58.102 & $+03: 27: 56.473$ & $16.538(012)$ & $.045(005)$ & $15.200(005)$ & $14.758(005)$ & $14.216(006)$ & $15.609(004)$ & $14.914(004)$ & $14.662(004)$ \\
\hline 4 & 01:20:02.123 & $+03: 22: 04.735$ & $16.183(0$ & 15.993 & 15.311 & $14.963(0$ & & 15.615 & 15.10 & \\
\hline 5 & $01: 20: 15.741$ & $+03: 27: 27.932$ & 14.593(007) & $14.396(004)$ & $13.664(004)$ & $13.539(013)$ & 13.904 & 14.000( & $13.434(004)$ & $14.888(012)$ \\
\hline 6 & .708 & $+03: 2$ & $.114(008)$ & 06) & $14.437(006)$ & $14.207(0$ & 14.040 & 14.685( & $14.275(004)$ & 14.597 \\
\hline 7 & 8.888 & $+03: 2$ & $17.790(021)$ & $17.635(007)$ & $16.934(007)$ & $16.574(007)$ & 16.102( & $17.250(005)$ & $16.720(005)$ & 16.53 \\
\hline 8 & 01:19:55.296 & $+03: 21: 52.628$ & $16.527(011)$ & $16.420(006)$ & $15.796(C$ & $15.478(0$ & 15.045 & $16.062(004)$ & $15.619(004)$ & $15.465(C$ \\
\hline 9 & $01: 20$ & $+03: 2$ & $17.123(015)$ & $17.203(007)$ & $16.646(007)$ & $16.369(007)$ & $83(009)$ & $16.869(005)$ & $16.503(005)$ & 16.3 \\
\hline 10 & 01:19: & $+03: 2$ & $15.444(009)$ & 15.191(006) & $14.508(006)$ & $14.230(005)$ & 14.000 & 14.812 & $14.302(004)$ & $14.597(003)$ \\
\hline 11 & $01: 20$ & $+03: 2$ & $16.748(012)$ & 16.1 & $15.351(006)$ & 14.9 & $14.420(006)$ & 15.750 & $15.073(004)$ & 14.858 \\
\hline 12 & 01:20:04.722 & $+03: 29: 32.716$ & $15.742(009)$ & $15.545(005)$ & $14.854(005)$ & $14.497(005)$ & 14.01 & 15.16 & 14.644( & $14.447(004)$ \\
\hline 13 & 01:19:57.199 & $+03: 27: 11.729$ & $17.634(018)$ & $17.533(007)$ & $16.874(007)$ & $16.532(007)$ & & $17.163(005)$ & $16.679(005)$ & $16.486(005)$ \\
\hline 14 & 01:20: & $+03: 2$ & & 16.4 & & & 14.9 & 16.0 & 15.5 & 15.3 \\
\hline 15 & 01:20: & $+03: 28: 34.205$ & $14.711(011)$ & $14.988(009)$ & $14.325(009)$ & $13.898(007)$ & $13.041(006)$ & $14.617(006)$ & $14.129(007)$ & $13.383(002)$ \\
\hline 16 & 01:20:53.340 & $+03: 16: 40.750$ & $13.768(009)$ & $12.763(001)$ & $12.248(001)$ & 11.994(001) & $12.186(009)$ & $12.444(001)$ & $12.126(001)$ & $12.026(001)$ \\
\hline 17 & 01:20:53.291 & $+03: 26: 42.976$ & $13.709(010)$ & $12.387(001)$ & $11.927(001)$ & $11.699(001)$ & $11.956(008)$ & $12.088(001)$ & $11.831(001)$ & $11.733(001)$ \\
\hline 18 & & $+03: 25: 52.392$ & $13.672(009)$ & $12.566(001)$ & & $11.913(001)$ & $12.068(008)$ & $12.275(001)$ & $12.045(001)$ & $11.949(001)$ \\
\hline 19 & 01:19:37.509 & $+03: 17: 06.781$ & $14.010(021)$ & $14.800(014)$ & $14.010(014)$ & $13.655(015)$ & $11.016(011)$ & $14.383(010)$ & $13.751(010)$ & $13.889(011)$ \\
\hline 20 & $01: 19: 56.437$ & $+03: 29: 39.264$ & $15.289(008)$ & $15.176(005)$ & $14.561(005)$ & $14.268(005)$ & $13.901(005)$ & $14.821(004)$ & $14.389(004)$ & $14.363(003)$ \\
\hline
\end{tabular}

${ }^{a}$ Standard stars used for calibration of instrumental magnitudes.

Table 2. Photometric Observations of SN 2017fgc by Ground-Based Telescopes

\begin{tabular}{|c|c|c|c|c|c|c|c|c|c|c|c|}
\hline MJD & Epoch $^{a}$ & $U$ (mag) & $B$ (mag) & $V(\mathrm{mag})$ & $R$ (mag) & $I$ (mag) & $g$ (mag) & $r$ (mag) & $i(\mathrm{mag})$ & Clear (mag) & Telescope \\
\hline 57947.10 & -12.30 & $15.304(100)$ & $15.446(043)$ & $15.381(040)$ & $\cdots$ & $\cdots$ & $15.297(033)$ & $15.223(033)$ & $15.742(048)$ & $\cdots$ & LCO \\
\hline 57948.16 & -11.24 & $14.743(076)$ & $15.010(034)$ & $14.928(034)$ & $\ldots$ & $\ldots$ & $14.906(027)$ & $14.857(027)$ & $15.356(039)$ & $\ldots$ & LCO \\
\hline 57954.72 & -4.68 & $13.635(110)$ & $13.948(051)$ & $13.932(051)$ & $\ldots$ & $\ldots$ & $13.893(056)$ & $13.874(037)$ & $14.420(063)$ & $\cdots$ & LCO \\
\hline 57955.81 & -3.59 & $13.571(052)$ & $13.875(024)$ & $13.820(022)$ & $\ldots$ & $\ldots$ & $13.804(019)$ & $13.868(021)$ & $14.345(030)$ & $\ldots$ & LCO \\
\hline 57956.81 & -2.59 & $13.527(045)$ & $13.838(019)$ & $13.673(017)$ & $\ldots$ & $\ldots$ & $13.716(015)$ & $13.609(015)$ & $14.316(023)$ & $\ldots$ & LCO \\
\hline 57957.94 & -1.46 & $13.545(054)$ & $13.811(024)$ & $13.699(022)$ & $\ldots$ & $\ldots$ & $13.711(018)$ & $13.644(019)$ & $14.319(030)$ & $\ldots$ & LCO \\
\hline 57959.49 & +0.09 & $\ldots$ & $13.793(034)$ & $13.596(030)$ & $13.522(020)$ & $13.889(031)$ & $\ldots$ & $\ldots$ & $\ldots$ & $\ldots$ & Nickel \\
\hline 57959.82 & +0.42 & $13.552(064)$ & $13.801(028)$ & $13.655(024)$ & $\ldots$ & $\ldots$ & $13.688(023)$ & $13.572(022)$ & $14.337(054)$ & $\cdots$ & LCO \\
\hline 57960.49 & +1.09 & $\ldots$ & $13.819(065)$ & $13.548(036)$ & $13.566(048)$ & $14.038(055)$ & $\ldots$ & $\ldots$ & $\ldots$ & $13.609(054)$ & KAIT4 \\
\hline 57961.49 & +2.09 & $\ldots$ & $\ldots$ & $\ldots$ & $\ldots$ & $\ldots$ & $\ldots$ & $\cdots$ & $\ldots$ & $13.589(051)$ & KAIT4 \\
\hline$\vdots$ & $\vdots$ & $\vdots$ & $\vdots$ & $\vdots$ & $\vdots$ & & $\vdots$ & $\vdots$ & & $\vdots$ & $\vdots$ \\
\hline 58134.25 & +174.85 & $\cdots$ & $18.326(128)$ & $18.276(101)$ & $18.973(134)$ & 19.131(196) & $\ldots$ & $\cdots$ & $\cdots$ & $\ldots$ & TNT \\
\hline 58136.10 & +176.70 & $\ldots$ & $\ldots$ & $\ldots$ & $\ldots$ & $\ldots$ & $\ldots$ & $\ldots$ & $\cdots$ & $18.341(135)$ & KAIT4 \\
\hline 58137.25 & +177.85 & $\ldots$ & $18.408(112)$ & $18.159(086)$ & $18.790(104)$ & $19.342(174)$ & $\ldots$ & $\ldots$ & $\ldots$ & $\ldots$ & TNT \\
\hline 58144.11 & +184.71 & $\ldots$ & $18.659(222)$ & $18.446(136)$ & $\ldots$ & $\ldots$ & $18.005(119)$ & $19.192(102)$ & $19.205(186)$ & $\ldots$ & LCO \\
\hline 58146.14 & +186.74 & $\ldots$ & $\ldots$ & $\ldots$ & $\ldots$ & $\ldots$ & $\ldots$ & $\ldots$ & $\ldots$ & $18.629(169)$ & KAIT4 \\
\hline 58150.11 & +190.71 & $\cdots$ & $\ldots$ & $\ldots$ & $\ldots$ & $\ldots$ & $\ldots$ & $\cdots$ & $\cdots$ & $18.427(169)$ & KAIT4 \\
\hline 58152.25 & +192.85 & $\ldots$ & $18.195(147)$ & $18.446(108)$ & $19.218(159)$ & $\ldots$ & $\ldots$ & $\ldots$ & $\cdots$ & $\ldots$ & TNT \\
\hline
\end{tabular}

Table 2 continued 
OBSERVATIONS OF SN 2017FGC

Table 2 (continued)

\begin{tabular}{|c|c|c|c|c|c|c|c|c|c|c|c|}
\hline MJD & $\operatorname{Epoch}^{a}$ & $U$ (mag) & $B$ (mag) & $V$ (mag) & $R$ (mag) & $I$ (mag) & $g(\mathrm{mag})$ & $r(\mathrm{mag})$ & $i(\mathrm{mag})$ & Clear (mag) & Telescope \\
\hline 58154.12 & +194.72 & $\cdots$ & $18.444(116)$ & $18.660(182)$ & $19.690(315)$ & $19.112(242)$ & $\cdots$ & $\cdots$ & $\cdots$ & 18.634(076) & KAIT4 \\
\hline 58162.12 & +202.72 & $\cdots$ & $\ldots$ & $\ldots$ & $\ldots$ & $\ldots$ & $\cdots$ & $\cdots$ & $\cdots$ & $18.802(128)$ & KAIT4 \\
\hline 58164.12 & +204.72 & $\ldots$ & $\ldots$ & $\ldots$ & $\ldots$ & $\ldots$ & $\ldots$ & $\ldots$ & $\ldots$ & $18.746(136)$ & KAIT4 \\
\hline
\end{tabular}

${ }^{a}$ Relative to the epoch of $B$-band maximum brightness (MJD $\left.=57,959.4\right)$. Measurements are calibrated to the AB magnitude system. A machine-readable file is available for this table. 
Table 3. Spectroscopic Observations of SN 2017fgc

\begin{tabular}{rrrrr}
\hline \hline MJD & Epoch $^{a}$ & $\lambda_{\text {Start }}$ & \multicolumn{1}{c}{$\lambda_{\text {End }}$} & Telescope \\
\hline 57947.7 & -11.7 & 3231 & 9918 & LCO \\
57952.5 & -6.9 & 3616 & 10,400 & Lick 3 m \\
57952.6 & -6.8 & 3226 & 9919 & LCO \\
57959.5 & -0.1 & 3226 & 9919 & LCO \\
57960.5 & +1.1 & 3622 & 10,400 & Lick 3 m \\
57961.5 & +2.1 & 3226 & 9919 & LCO \\
57964.5 & +5.1 & 3620 & 10,400 & Lick 3 m \\
57966.5 & +7.0 & 3620 & 10,400 & Lick 3 m \\
57966.5 & +7.1 & 3226 & 9920 & LCO \\
57972.6 & +13.2 & 3226 & 9919 & LCO \\
57981.5 & +21.1 & 3231 & 9919 & LCO \\
57987.5 & +28.4 & 3473 & 9099 & LJT \\
57988.7 & +29.3 & 3180 & 9919 & LCO \\
57992.5 & +33.1 & 3622 & 10,400 & Lick 3 m \\
57997.7 & +38.2 & 3374 & 9919 & LCO \\
58006.6 & +47.2 & 3972 & 9919 & LCO \\
58010.5 & +51.1 & 3632 & 10,400 & Lick 3 m \\
58011.5 & +52.1 & 3634 & 10,400 & Lick 3 m \\
58013.6 & +54.2 & 3226 & 9919 & LCO \\
58023.4 & +64.0 & 3277 & 9918 & LCO \\
58023.5 & +64.1 & 3632 & 10,400 & Lick 3 m \\
58023.6 & +64.2 & 3276 & 9919 & LCO \\
58041.5 & +82.1 & 3474 & 9224 & LCO \\
58045.4 & +86.0 & 3620 & 10,400 & Lick 3 m \\
58051.4 & +92.0 & 3620 & 10,400 & Lick 3 m \\
58053.1 & +93.7 & 3701 & 8747 & XLT \\
58056.4 & +97.0 & 3622 & 10,398 & Lick 3 m \\
58062.5 & +103.1 & 3276 & 9919 & LCO \\
58083.1 & +123.7 & 3725 & 8739 & XLT \\
58091.2 & +131.8 & 3176 & 9918 & LCO \\
58101.2 & +141.8 & 3227 & 9919 & LCO \\
58108.0 & +148.6 & 5665 & 6963 & LCO \\
58109.1 & +149.7 & 2990 & 24,790 & ESO public \\
58116.3 & +156.9 & 3178 & 9902 & LCO \\
58131.2 & +171.8 & 3226 & 9225 & LCO \\
58343.3 & +180.9 & 5711 & 7002 & LCO \\
\hline$a$ & +383.9 & 2990 & 24,790 & ESO public \\
\hline & +388.9 & 2990 & 24,790 & ESO public \\
\hline & & & & \\
5819 &
\end{tabular}

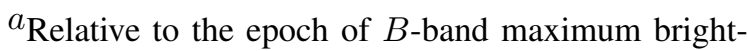
ness (MJD $=57,959.4)$. 
Table 4. DLT40 Photometry of SN 2017fgc in the Clear Band

\begin{tabular}{crrr}
\hline \hline MJD & Epoch $^{a}$ & \multicolumn{1}{c}{ Clear (mag) } & Telescope \\
\hline 57942.3 & -17.1 & $19.183(\cdots)$ & Prompt5 \\
57943.3 & -16.1 & $17.324(086)$ & Prompt5 \\
57944.4 & -15.0 & $16.550(040)$ & Prompt5 \\
57945.3 & -14.1 & $16.096(030)$ & Prompt5 \\
57946.4 & -13.0 & $15.532(020)$ & Prompt5 \\
57947.3 & -12.1 & $15.196(027)$ & Prompt5 \\
57952.3 & -7.1 & $14.130(016)$ & Prompt5 \\
57953.3 & -6.1 & $14.015(016)$ & Prompt5 \\
57955.3 & -4.1 & $13.854(016)$ & Prompt5 \\
57956.3 & -3.1 & $13.779(016)$ & Prompt5 \\
$\vdots$ & $\vdots$ & & $\vdots$ \\
58098.1 & 138.7 & $17.728(056)$ & Prompt5 \\
58099.1 & 139.7 & $17.830(064)$ & Prompt5 \\
58100.1 & 140.7 & $17.687(060)$ & Prompt5 \\
58101.1 & 141.7 & $17.814(057)$ & Prompt5 \\
58102.1 & 142.7 & $17.961(063)$ & Prompt5 \\
58103.1 & 143.7 & $17.857(060)$ & Prompt5 \\
58108.1 & 148.7 & $18.137(075)$ & Prompt5 \\
58110.1 & 150.7 & $17.998(070)$ & Prompt5 \\
58111.6 & 152.2 & $18.123(067)$ & Meckering \\
58124.5 & 165.1 & $18.593(079)$ & Meckering \\
\hline
\end{tabular}

${ }^{a}$ Relative to the epoch of $B$-band maximum (MJD $=57,959.4$ ). A machine-readable file is available for this table. 
Table 5. Parameters of SN $2017 \mathrm{fgc}$

\begin{tabular}{lc}
\hline \multicolumn{1}{c}{ Parameter } & \multicolumn{1}{c}{ Value } \\
\hline$B_{\max }$ & $13.07 \pm 0.11 \mathrm{mag}$ \\
$B_{\max }-V_{\max }$ & $0.16 \pm 0.13 \mathrm{mag}$ \\
$M_{\max }(B)$ & $-19.32 \pm 0.13 \mathrm{mag}$ \\
$E(B-V)_{\text {host }}$ & $0.17 \pm 0.07 \mathrm{mag}$ \\
$\Delta m_{15}(B)$ & $1.05 \pm 0.07 \mathrm{mag}$ \\
$s_{B V}$ & $1.19 \pm 0.03$ \\
$t_{\text {max }}(B)$ & $57,959.4 \pm 0.4 \mathrm{~d}$ \\
$t_{0}$ & $57941.4 \pm 0.4 \mathrm{~d}$ \\
$\tau_{\text {rise }}$ & $18.0 \pm 0.4 \mathrm{~d}$ \\
$L_{\mathrm{bol}}^{\max }$ & $(1.32 \pm 0.13) \times 10^{43} \mathrm{erg} \mathrm{s}^{-1}$ \\
$M_{56} \mathrm{Ni}$ & $0.51 \pm 0.03 M_{\odot}$ \\
$v_{0}(\mathrm{Si} \mathrm{II})$ & Spectroscopic \\
$\dot{v}(\mathrm{Si}$ II $)$ & $15,000 \pm 150 \mathrm{~km} \mathrm{~s}^{-1}$ \\
$R(\mathrm{Si} \mathrm{II})$ & $120 \pm 10 \mathrm{~km} \mathrm{~s}^{-1} \mathrm{~d}^{-1}$ \\
\hline
\end{tabular}

